\title{
Visuomotor brain network activation and functional connectivity among individuals with autism spectrum disorder
}

\author{
Rebecca J. Lepping ${ }^{1}$ (1) | Walker S. McKinney ${ }^{2}$ ( ) | Grant C. Magnon ${ }^{3}$ | \\ Sarah K. Keedy ${ }^{4}$ | Zheng Wang ${ }^{5,6}$ | Stephen A. Coombes ${ }^{6}$ | \\ David E. Vaillancourt $^{6}$ () | John A. Sweeney ${ }^{7}$ | Matthew W. Mosconi ${ }^{2}$
}

${ }^{1}$ Hoglund Biomedical Imaging Center, University of Kansas Medical Center, Kansas City, Kansas, USA

${ }^{2}$ Schiefelbusch Institute for Life Span Studies, Clinical Child Psychology Program, and Kansas Center for Autism Research and Training (K-CART), University of Kansas, Lawrence, Kansas, USA

${ }^{3}$ Department of Medicine, University of Pittsburgh School of Medicine, Pittsburgh, Pennsylvania, USA

${ }^{4}$ Department of Psychiatry and Behavioral Neuroscience, University of Chicago, Chicago, Illinois, USA

${ }^{5}$ Department of Occupational Therapy, University of Florida, Gainesville, Florida, USA

${ }^{6}$ Department of Applied Physiology and Kinesiology, University of Florida, Gainesville, Florida, USA

${ }^{7}$ Department of Psychiatry and Behavioral Neuroscience, University of Cincinnati College of Medicine, Cincinnati, Ohio, USA

\section{Correspondence}

Matthew W. Mosconi, Dole Human Development Center, 1000 Sunnyside Ave. Office 2018, Lawrence, KS 66045, USA.

Email: mosconi@ku.edu

\section{Funding information}

Kansas Center for Autism Research and Training (K-CART) Research Investment Council Strategic Initiative Grant; National Institutes of Health, Grant/Award Numbers: K23 MH092696, P50 ACE HD055751, R01 MH112734, R21 AG065621, U54 HD090216; University of Florida, Grant/Award Number: CTSI Pilot Award UL1TR001427

\begin{abstract}
Sensorimotor abnormalities are common in autism spectrum disorder (ASD) and predictive of functional outcomes, though their neural underpinnings remain poorly understood. Using functional magnetic resonance imaging, we examined both brain activation and functional connectivity during visuomotor behavior in 27 individuals with ASD and 30 typically developing (TD) controls (ages 9-35 years). Participants maintained a constant grip force while receiving visual feedback at three different visual gain levels. Relative to controls, ASD participants showed increased force variability, especially at high gain, and reduced entropy. Brain activation was greater in individuals with ASD than controls in supplementary motor area, bilateral superior parietal lobules, and contralateral middle frontal gyrus at high gain. During motor action, functional connectivity was reduced between parietal-premotor and parietalputamen in individuals with ASD compared to controls. Individuals with ASD also showed greater age-associated increases in functional connectivity between cerebellum and visual, motor, and prefrontal cortical areas relative to controls. These results indicate that visuomotor deficits in ASD are associated with atypical activation and functional connectivity of posterior parietal, premotor, and striatal circuits involved in translating sensory feedback information into precision motor behaviors, and that functional connectivity of cerebellar-cortical sensorimotor and nonsensorimotor networks show delayed maturation.
\end{abstract}

\section{KEYWORDS}

autism spectrum disorder, fMRI, functional connectivity, motor function, visuomotor integration

This is an open access article under the terms of the Creative Commons Attribution-NonCommercial-NoDerivs License, which permits use and distribution in any medium, provided the original work is properly cited, the use is non-commercial and no modifications or adaptations are made.

(c) 2021 The Authors. Human Brain Mapping published by Wiley Periodicals LLC. 


\section{1 | INTRODUCTION}

Autism spectrum disorder (ASD) affects the development of multiple cognitive and behavioral abilities. The diverse range of clinical issues associated with ASD hinders progress identifying neurodevelopmental mechanisms. Sensorimotor behaviors represent a promising target for advancing knowledge of neurodevelopmental mechanisms of ASD because they are frequently disrupted (Coll, Foster, Meilleur, Brambati, \& Hyde, 2020), predictive of worse outcomes (Bal et al., 2020; Nebel et al., 2016), familial (Mosconi et al., 2010; Schmitt et al., 2019), and supported by well understood and highly specialized brain networks. Quantitative studies of sensorimotor behavior and brain function in ASD may advance a more mechanistic understanding of the disorder.

Individuals with ASD show a range of sensorimotor abnormalities including repetitive motor mannerisms (American Psychiatric Association, 2013), less precise saccadic eye movements (Johnson, Rinehart, White, Millist, \& Fielding, 2013; Schmitt, Cook, Sweeney, \& Mosconi, 2014; Takarae, Minshew, Luna, \& Sweeney, 2004), increased postural sway (Bojanek, Wang, White, \& Mosconi, 2020; Wang et al., 2016), and dyspraxia (MacNeil \& Mostofsky, 2012). Multiple studies converge to suggest that individuals with ASD show reduced ability to integrate visual or multisensory feedback during motor behavior (Haswell, Izawa, Dowell, Mostofsky, \& Shadmehr, 2009; Sharer et al., 2015). Consistent with this hypothesis, we have found that individuals with ASD show greater motor variability and reduced entropy during visually guided precision gripping implicating deficient visual feedback control of motor behavior (Mosconi et al., 2015; Neely et al., 2019; Wang et al., 2016).

During visually guided motor behavior (i.e., visuomotor behavior), visual feedback is processed in visual and posterior parietal cortices, including superior (SPL) and inferior parietal lobules (IPL), which project to premotor and primary motor cortices (M1; Caminiti, Ferraina, \& Johnson, 1996; Vaillancourt, Mayka, \& Corcos, 2006). Visuomotor behavior is supported by a subcortical circuit in which feedback information is relayed from posterior parietal cortex to cerebellum where error information is translated into reactive motor commands to M1 (Glickstein, 2000; Glover, Wall, \& Smith, 2012; Stein \& Glickstein, 1992). Posterior cerebellum and basal ganglia, including putamen and caudate, are involved in modulating motor output timing and amplitude (Prodoehl, Yu, Wasson, Corcos, \& Vaillancourt, 2008; Spraker et al., 2012). The function of these networks is consolidated throughout childhood and early adulthood as evidenced by strengthening of long-distance cerebellar-cortical pathways and weakening of local functional circuits (Amemiya et al., 2019). Developmental studies of visuomotor network function in ASD identified increases in cerebellar-cortical functional connectivity in childhood (Stoodley et al., 2017) that become more severe during adolescence and adulthood (Holiga et al., 2019). Despite evidence that task-based functional magnetic resonance imaging ( $\mathrm{fMRI}$ ) approaches to assessing functional connectivity provide more robust predictors of behavioral trait dimensions than resting state (Greene, Gao, Scheinost, \& Constable, 2018), few studies have assessed task-dependent functional connectivity of visuomotor networks in ASD or their variance across childhood and adulthood.

Several studies have assessed visuomotor network activation in ASD. Takarae, Minshew, Luna, and Sweeney (2007) reported reduced frontal and parietal eye field and cerebellar activation during saccades in adults with ASD relative to typically developing (TD) controls. Individuals with ASD also showed greater activity in frontal, striatal, and cerebellar regions suggesting increased involvement of cognitive control networks. Finger tapping studies have also documented aberrant frontal, parietal and cerebellar activity in ASD (Allen \& Courchesne, 2003; Allen, Muller, \& Courchesne, 2004; Mostofsky et al., 2009; Muller, Kleinhans, Kemmotsu, Pierce, \& Courchesne, 2003; Muller, Pierce, Ambrose, Allen, \& Courchesne, 2001). In the one known study to assess functional connectivity during motor behavior, Mostofsky et al. (2009) documented reduced cerebellar-thalamo-cortical functional connectivity alongside increased supplementary motor area activation and reduced cerebellar activation in ASD during finger tapping. While finger-tapping studies have been important for identifying gross motor impairment, studies of fine motor control, such as during precision gripping, allow for interrogation of the visuomotor system in a way that more accurately represents functional motor behaviors. We previously have described fMRI task activation increases in ventral premotor cortex and cerebellum during a precision gripping task in ASD (Unruh et al., 2019). While the majority of motor behaviors are guided by visual feedback, and visuomotor behaviors are impaired in ASD, no known fMRI studies have examined brain network function and connectivity in ASD during visually guided precision motor behavior.

Using fMRI, we assessed visuomotor network activation and functional connectivity during precision gripping. We predicted that, relative to TD controls, individuals with ASD would show increased grip force variability and reduced force entropy. Based on prior behavioral findings that visuomotor impairments in ASD are more severe at high visual feedback gain (Mosconi et al., 2015), we tested behavior and brain function across three visual gain levels. We predicted that increases in force variability and reductions in entropy would be more severe when visual feedback was amplified indicating deficits processing increased sensory information. Consistent with the overarching hypothesis that visuomotor deficits in ASD reflect alterations in dynamically adjusting motor behavior in response to sensory feedback, we predicted individuals with ASD would show reduced activation of visual cortex, posterior parietal cortex, and cerebellum during visuomotor control relative to TD controls. We also expected reduced parietal-cerebellar, parietal-striatal, and parietal-motor cortex functional connectivity in ASD during visuomotor behavior. Individuals were studied across a wide age range (9-35 years) so that ageassociated differences in visuomotor behavior and brain network function could be examined. Consistent with prior resting state fMRI studies (Padmanabhan, Lynn, Foran, Luna, \& O'Hearn, 2013), we predicted visuomotor brain dysfunctions relative to TD controls would be more severe at older ages. 


\section{2 | MATERIALS AND METHODS}

\section{1 | Participants}

Twenty-seven participants with ASD and 30 TD controls were recruited from the community, and group-matched on age (range: 935 years), nonverbal IQ, and handedness (Table 1). IQ was assessed using the Wechsler Abbreviated Scale of Intelligence (WASI; Wechsler, 1999). ASD diagnoses were confirmed using the Autism Diagnostic Inventory-Revised (ADI-R; Lord, Rutter, \& Le Couteur, 1994), the Autism Diagnostic Observation Schedule (ADOS2; Lord et al., 1989) and expert clinical opinion using DSM-IV-TR criteria (American Psychiatric Association, 2000). Participants with ASD were excluded if they had a known genetic or metabolic disorder associated with ASD (e.g., fragile $X$ syndrome, tuberous sclerosis). TD control participants were excluded if they had ever been diagnosed with ASD, or reported current or past psychiatric or neurological disorders, family history of ASD in first-, second- or third-degree relatives, or first-degree relative with a developmental or learning disorder, psychosis, or obsessive-compulsive disorder. No participants were taking medications known to affect motor control, including antipsychotics, stimulants, or anticonvulsants (Reilly, Harris, Keshavan, \& Sweeney, 2005), nor had a history of head injury, birth injury, or seizure disorder. Participants' uncorrected far visual acuity was at least 20/40. Written informed consent was obtained for adult participants and parental consent was obtained for participants younger than 18 years. Minors provided written assent. Study procedures were approved by the local Institutional Review Board and abided by the Code of Ethics of the World Medical Association (Declaration of Helsinki).

\section{2 | Data acquisition}

\subsection{1 | Visuomotor behavior data acquisition}

Participants used their right thumb and index finger to exert opposing forces on a custom fiber-optic force transducer (Figure 1a; Neuroimaging Solutions), constructed from rigid, nonmetallic material to ensure safety and consistent linearity, sensitivity, and accuracy inside the MR environment. The force signal was transmitted from the transducer via fiber-optic cable to a si425 Optical Sensing Interrogator (Micron Optics), which digitized the force data at $125 \mathrm{~Hz}$. Customized software written in LabVIEW (National Instruments, Austin, TX) converted analog force data to Newtons $(N)$ at a resolution of $0.025 \mathrm{~N}$. Output from the force transducer was presented to the participant using a visual display through a projection system placed on the head coil and a mirror located $35 \mathrm{~cm}$ from the participant's eyes inside the MR environment (resolution: $640 \times 480$ pixels; refresh rate: $60 \mathrm{~Hz})$.

TAB LE 1 Demographic characteristics for individuals with ASD and TD controls

\begin{tabular}{|c|c|c|c|c|}
\hline & $\operatorname{ASD}(N=27)$ & TD controls $(N=30)$ & $t$ & $p$ \\
\hline Age (years) & $18.4 \pm 6.7(9-35)$ & $18.9 \pm 7.0(10-35)$ & 0.25 & .804 \\
\hline Sex (\% male) & $88.9 \%$ & $60.0 \%$ & $6.12^{\mathrm{a}}$ & $.013^{*}$ \\
\hline Handedness (\% right-handed) & $92.6 \%$ & $86.7 \%$ & $1.07^{\mathrm{a}}$ & .587 \\
\hline Race & & & $5.21^{\mathrm{a}}$ & .266 \\
\hline \% White & $88.9 \%$ & $83.3 \%$ & - & - \\
\hline$\%$ Black & $3.7 \%$ & $6.7 \%$ & - & - \\
\hline \% American Indian or Alaska Native & $3.7 \%$ & $0 \%$ & - & - \\
\hline$\%$ Other or unknown & $3.7 \%$ & $0 \%$ & - & - \\
\hline Ethnicity & & & 4.28 & .233 \\
\hline \% Hispanic/Latinx & $14.8 \%$ & $20 \%$ & - & - \\
\hline Verbal IQ & $102.0 \pm 19.7(74-141)$ & $112.7 \pm 15.6(86-149)$ & 2.14 & $.038^{*}$ \\
\hline Nonverbal IQ & $100.8 \pm 19.6(59-127)$ & $107.8 \pm 13.1(78-143)$ & 1.47 & .148 \\
\hline ADI-R (A) & $19.9 \pm 5.7(7-28)$ & - & - & - \\
\hline ADI-R (verbal B) & $16.8 \pm 3.8(10-24)$ & - & - & - \\
\hline ADI-R (C) & $5.7 \pm 2.4(3-11)$ & - & - & - \\
\hline ADOS-2 (total CSS) & $7.0 \pm 2.2(3-10)$ & - & - & - \\
\hline Right hand MVC (Newtons) & $73.9 \pm 19.6(40-110)$ & $63.3 \pm 15.4(40-110)$ & -1.60 & .112 \\
\hline
\end{tabular}

Note: Values reported as mean $\pm S D$ (range).

Abbreviations: ADI, Autism Diagnostic Interview-Revised; ADOS, Autism Diagnostic Observation Schedule; CSS, Calculated Severity Score; IQ, intelligence quotient.

${ }^{a}$ Chi-square statistic. ${ }^{*} p<.05$. 


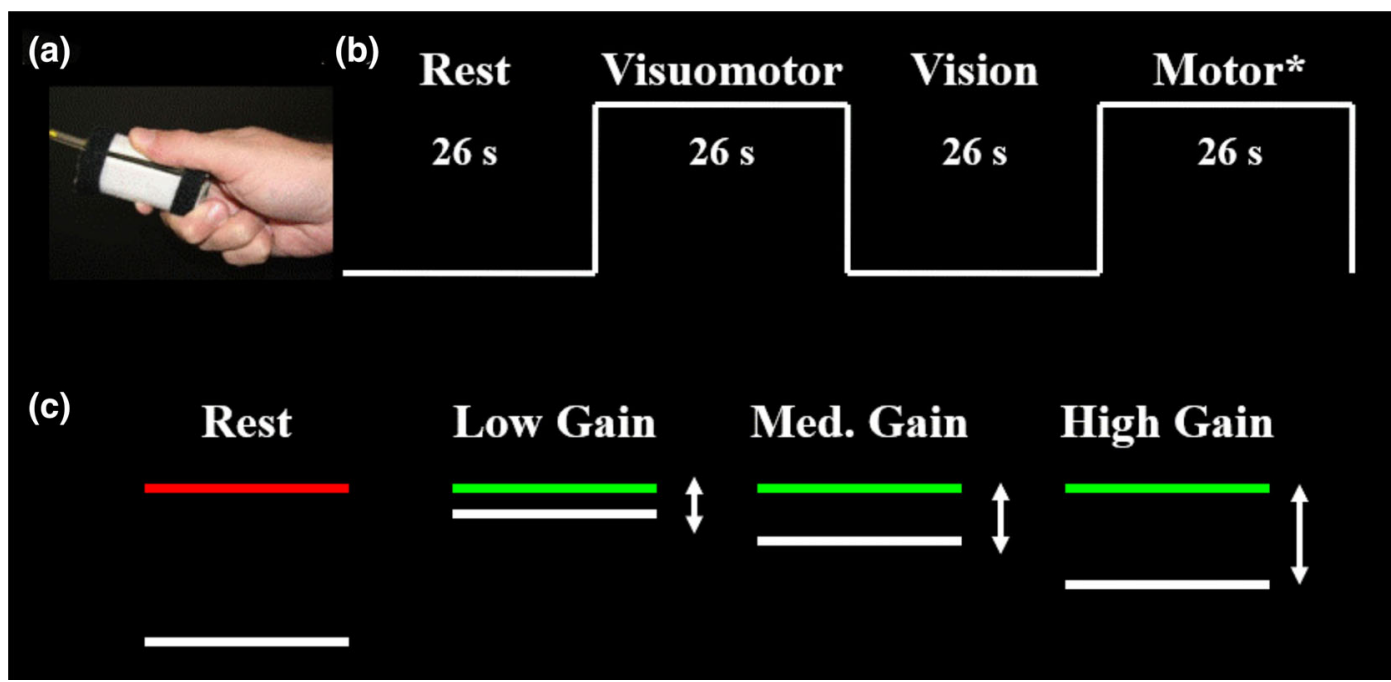

FIG URE 1 (a) Grip configuration and force transducer. (b) Schematic representation of task condition blocks. Blocks are $26 \mathrm{~s}$ in duration. Each condition was repeated three times at each gain level, ending each run with a rest block. (c) Schematic representation of the visuomotor task. The red target bar turned green to cue the beginning of each trial. The white force bar moved upwards with increased force. The force bar traveled a greater distance per change in force for higher relative to lower gains. *Results from the motor only condition in which no visual feedback was provided are not reported due to participants' difficulties remembering to press

\subsection{2 | MRI data acquisition}

MR scans were performed using a 3 Tesla scanner (General Electric Medical Systems, Milwaukee, WI) with a standard birdcage quadrature single channel head coil. Participants lay supine and their heads were stabilized using adjustable padding. Functional images were acquired using a T2* single shot gradient-echo echo-planar pulse sequence: repetition time $(T R)=2000 \mathrm{~ms}$, echo time $(T E)=25 \mathrm{~ms}$, flip angle $=90^{\circ}$, in-plane resolution $=3 \times 3 \mathrm{~mm}, 64 \times 64$ acquisition matrix, field of view $(F O V)=200 \mathrm{~mm}, 33$ axial slices, $3 \mathrm{~mm}$ thickness with $1 \mathrm{~mm}$ gap. An anatomical scan was acquired using a T1-weighted 3D inversion recovery fast spoiled gradient recalled pulse (SPGR) sequence: $\mathrm{TR}=25 \mathrm{~ms}$, TE $=3 \mathrm{~ms}$, flip angle $=90^{\circ}$, in-plane resolution $=0.9 \times 0.9 \mathrm{~mm}, 256 \times 192$ acquisition matrix, FOV $=24 \times 24,120$ axial slices, $1.5 \mathrm{~mm}$ thickness with no gap. Both sequences covered the entire brain.

\subsection{Experimental design}

Participants completed a 30-min pre-fMRI training session completing at least one run of the fMRI task. The training session ensured participants understood the instructions and were able to complete the task without verbal cues, and also helped eliminate the transitory period of motor learning (Coombes, Corcos, Sprute, \& Vaillancourt, 2010). Each participant's maximum voluntary contraction (MVC) was calculated before beginning the $\mathrm{fMRI}$ experiment. Participants produced their maximum force against a strain-gauge dynamometer over three trials (Sammons Preston, Rolyan, Bolingbrook, IL). MVC was estimated as the mean maximum value (Mosconi et al., 2015; Vaillancourt, Mayka, \& Corcos, 2006).
Three fMRI task runs were administered. During each run, participants completed four task conditions with their right hand in the following order: (1) rest, (2) visuomotor, (3) vision, and (4) motor only. Each condition block was $26 \mathrm{~s}$ long. The condition series was administered three times, with an additional rest block at the end of each run (e.g., $R-V M-V-M-R-V M-V-M-R-V M-V-M-R$; Figure $1 b)$. The duration of each run was 5:38. Each of the three runs followed the same sequence, but the visual gain of feedback was varied. The target force was fixed at $15 \%$ of the MVC. During the rest condition, participants viewed a horizontal white force bar and a parallel red target bar (Figure 1b,c). They were instructed to watch the static bars, and ensure they did not press the transducer. During the visuomotor condition, the red target bar turned green at trial onset, and the white force bar moved upwards with increased force and downward with decreased force. Participants were instructed to press when the red target bar turned green, and to keep pressing so that the white force bar stayed as steady as possible matching the green target bar. Vision only trials were administered to assess blood oxygenation level dependent (BOLD) responses to visual motion without force production. Participants viewed the green target bar and the moving force bar, but did not produce force. The force bar oscillated around the target bar, and participants were instructed to watch the screen. The oscillation frequency of the force bar was a $1-\mathrm{Hz}$ sine wave with a small amount of white noise. The amplitude of oscillation matched the visual gain of the run being administered. The motor only condition was administered to assess BOLD responses to motor behavior without visual feedback. During this condition, the target bar remained green, but the force bar disappeared after $1.5 \mathrm{~s}$. Participants were instructed to continue pressing with the same level of force used to reach the target. Due to participants' difficulties remembering to press during the motor only condition, these data are not reported here. 
Visual gain was manipulated by changing the visual angle. Visual angle $(\alpha)$ was varied by altering the height of force fluctuations $\left(\mathrm{H}_{1}\right)$ on the video display, relative to the distance between the participant's eyes and screen (D) using the following formula (Vaillancourt, Haibach, \& Newell, 2006):

$$
\alpha=2 * \tan ^{-1}\left(\mathrm{H}_{1} / \mathrm{D}\right)
$$

Based on prior studies, we assumed that participants produced force at $6 \mathrm{~N}$ with a SD of $0.3 \mathrm{~N}$ (Mosconi et al., 2015; Slifkin \& Newell, 1999). SD multiplied by six approximated the full range of estimated variance. $\mathrm{H}_{1}$ was varied to approximate three visual angles: $0.018^{\circ}$ (low gain), $0.192^{\circ}$ (medium gain), and $2.023^{\circ}$ (high gain) to obtain values above and below one degree (Vaillancourt, Mayka, \& Corcos, 2006). The order of administration of gain levels was counterbalanced across participants.

\section{4 | Data processing}

\subsection{1 | Visuomotor behavior data processing}

To examine visuomotor performance, the behavior time series data were processed using a custom LabVIEW program (National Instruments, Austin, TX) (Elliott, Vijayakumar, Zink, \& Hansen, 2007) and MATLAB (The MathWorks Inc., Natick, MA). Time series data for each fMRI task run were digitally filtered using a fourth-order Butterworth filter with a $30 \mathrm{~Hz}$ low-pass cutoff. To examine sustained force output, the first $2 \mathrm{~s}$ and last second of the force trace were excluded for each $26 \mathrm{~s}$ visuomotor block. The trace was then linearly detrended to account for changes in mean force over the course of the trial. Mean force was calculated as the average force output of the time series as a measure of individuals' ability to complete the task. The within-trial $S D$ of the force time series was calculated to examine the amplitude of performance variability. To examine the time-dependent structure of the data, approximate entropy (ApEn) was calculated for each trial (Pincus \& Goldberger, 1994; Vaillancourt, Slifkin, \& Newell, 2001). ApEn reflects the predictability of future values based on previous values. For example, a sine wave has accurate short- and long-term predictability and corresponds to an ApEn near zero. Increases in signal complexity, reflective of the independence of each force value, returns an ApEn near two (e.g., white noise). The same algorithm and parameter settings ( $m=2 ; r=.2^{*} S D$ of the signal) were applied as in our previous work (McKinney et al., 2019; Mosconi et al., 2015; Slifkin, Vaillancourt, \& Newell, 2000; Unruh et al., 2019).

\subsection{2 $\quad$ fMRI data processing}

Imaging data were processed using the Analysis of Functional Neuroimages software (AFNI; https://afni.nimh.nih.gov; Cox, 1996). MR data were rejected for head movement artifact and failure to comply with task instructions for seven participants' low gain condition (ASD: 4, TD: 3), four participants' medium gain condition (ASD: 3, TD: 1), and six participants' high gain condition (ASD: 4, TD: 2). Participants were included in group analyses if they successfully completed at least one gain condition. Anatomical images were skullstripped and nonlinearly warped to Montreal Neurologic Institute (MNI) standard space (Fonov et al., 2011). Functional preprocessing steps followed our previously reported work (McKinney et al., 2020; Unruh et al., 2019). Slice-timing correction was applied. Consecutive volumes with $>0.5 \mathrm{~mm}$ framewise displacement were censored. Motion censoring data are in Table S1. The percent of TRs censored (gain effect: $F_{(2,95.21)}=0.74, p=.48$ ) and average motion per TR after censoring (gain effect: $F_{(2,95.64)}=2.48, p=.09$ ) were similar across gain levels and groups (percent of TRs censored group effect: $F_{(1,51.78)}$ $=2.05, p=.16$; average motion per TR group effect: $F_{(1,51.91)}=1.48$, $p=.23)$. Group differences did not vary as a function of gain for either percent of TRs censored (group $\times$ gain interaction: $F_{(2,95.21)}$ $=0.31, p=.73$ ) nor average motion per TR (group $\times$ gain interaction: $\left.F_{(2,95.63)}=0.74, p=.48\right)$.

Remaining functional volumes were rigidly aligned with anatomical data referenced to the minimum outlier volume and warped into standard space. Volumes were spatially smoothed to a $6 \mathrm{~mm}$ fullwidth half-maximum Gaussian kernel and scaled to the mean voxel time-series value of 100 . Functional data were regressed using a block function with six motion parameters ( $X, Y, Z$, roll, pitch, yaw) included as nuisance regressor terms. Regression outcomes represent the percent signal change $(\beta)$ of each contrast of interest (visuomotor-rest; vision-rest; visuomotor-vision) and associated $t$-statistics.

Separate psychophysiological interaction (PPI), or task-dependent functional connectivity analyses (Cisler, Bush, \& Steele, 2014; McLaren, Ries, Xu, \& Johnson, 2012) were conducted for eight hypothesis-driven seed regions of interest (ROIs) identified from previous precision gripping studies (Spraker et al., 2012; Vaillancourt, Mayka, \& Corcos, 2006), including bilateral IPL, SPL, cerebellar Crus I, and cerebellar lobules V/VI (Figure S1). Cortical ROls were obtained using Brainnetome, a cortical atlas parcellated from resting-state functional connectivity data of 40 healthy, right-handed adults (Fan et al., 2016). Cerebellar ROIs were obtained using the spatially unbiased infratentorial template (SUIT) cerebellar atlas (Diedrichsen, 2006), created by averaging high-resolution cerebellar/brainstem scans of 20 adults. The average time series of each seed ROI was calculated. A canonical hemodynamic response function (HRF) was calculated for visuomotor, vision, and rest blocks, as were interactions between the ROI time series and visuomotor, vision, and rest functions (i.e., PPI regressors). For each seed region, the seed time series, the $3 \mathrm{HRFs}$, the $3 \mathrm{PPI}$ regressors, and 12 motion regressors were included in regression models using AFNI's 3dDeconvolve program. Visuomotor-dependent functional connectivity for PPI contrasts of interest (visuomotor-rest) was calculated using AFNI's 3dcalc program.

\subsection{Statistical analysis}

Age, IQ, and MVC were compared between groups (ASD vs. TD controls) using two-sided independent samples $t$-tests. Handedness and 
sex were compared between groups using chi-square tests. Nonnormally distributed behavioral outcomes (force SD, ApEn) were logtransformed. A series of linear mixed effects models were performed to examine group differences in behavioral outcomes across visual gain levels (low, medium, and high). Mixed effects models were used to estimate missing data and model within subject variation in outcome measures. Age and sex were included as covariates in each model.

Group differences in brain activation and visuomotor-dependent functional connectivity were identified with linear mixed effects modeling using AFNI's 3dLME program (Chen, Saad, Britton, Pine, \& Cox, 2013). Separate 2 (group) $\times 3$ (gain) models with group $\times$ gain and group $\times$ age interaction terms were examined for visuomotorrest, vision-rest, and visuomotor-vision contrasts. Sex was included in each model as a covariate of no interest. A group-level mask (3dmask_tool) was applied to all 3dLME output to include voxels present in at least $50 \%$ of the data sets. Auto-correlation function (ACF) estimates derived from residual data were averaged across the sample and entered into AFNI's 3dClustSim program to estimate family-wise error correction at $\alpha<.05$. Based on this, we report significant clusters with at least 57 contiguous voxels $\left(1,539 \mathrm{~mm}^{3}\right)$ at voxel-wise $p<.005$ for group contrasts and group interactions. To better differentiate large clusters of activation observed in main effect tests of visual feedback gain, a more stringent voxel-wise threshold of $p<.001$ and 23 contiguous voxels $\left(621 \mathrm{~mm}^{3}\right)$ was used to achieve corrected $\alpha<.05$.

Linear mixed effects models were conducted to examine relationships between brain outcomes and behavioral and clinical variables. Maximum BOLD signal for individual participants for each significant cluster in group-contrast analyses was extracted using the clusters as masks. Comparison variables included force $S D$ and ApEn (both groups); and clinical measures (ASD only), including scores for the
ADOS (overall calculated severity score) and ADI (diagnostic algorithm scores for each subscale). Due to the exploratory nature of these analyses, we report as significant all relationships with uncorrected $p<.05$.

\section{3 | RESULTS}

\subsection{Visuomotor behavior performance}

Individuals with ASD and controls showed similar MVCs (Table 1; ASD: range: $40-110 \mathrm{~N}$; TD: range: $34-88 \mathrm{~N}$ ). Mean force was similar across gain levels (gain main effect: $F_{(2,104.31)}=0.06, p=.94$ ) and groups (Figure 2a; group main effect: $F_{(1,52.15)}=1.67, p=.20$ ). There was no interaction of group by gain level for mean force (group $\times$ gain interaction: $F_{(2,104.37)}=0.10, p=.91$ ). Mean force was greater in older participants (age main effect: $F_{(1,52.14)}=$ $11.14, p<.005)$, though age-associated increases in mean force were similar across groups (group $\times$ age interaction: $F_{(1,51.99)}=$ $0.61, p=.44)$.

Force $S D$ decreased with increases in visual gain (Figure $2 \mathrm{~b}$; gain main effect: $\left.F_{(2,103.82)}=80.10, p<.001\right)$. Individuals with ASD showed elevated force SD compared to TD controls (group main effect: $F_{(1,52.60)}=6.99, p=.01$; Figure $\left.2 b\right)$, and group differences varied as a function of gain (group $\times$ gain interaction: $F_{(2,103.90)}=3.39$, $p=.04)$. Post-hoc analyses revealed that individuals with ASD showed increased force variability compared to TD controls at high gain ( $\left.t_{98.3}=3.70, p<.005\right)$, but not at low $\left(t_{102.6}=1.41, p=.72\right)$ nor medium gain $\left(t_{106.7}=1.40, p=.73\right)$. Force $S D$ decreased as a function of increased age (age main effect: $F_{(1,52.54)}=5.70, p=.02$ ). Ageassociated differences were similar across groups (group $\times$ age interaction: $\left.F_{(1,52.33)}=0.13, p=.72\right)$. (a)

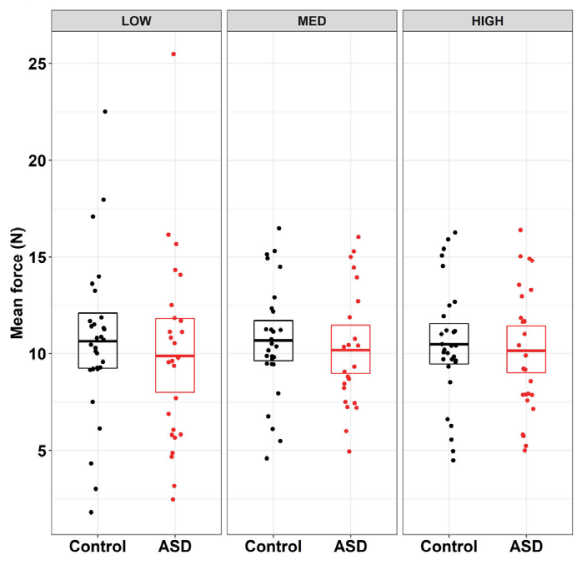

(b)

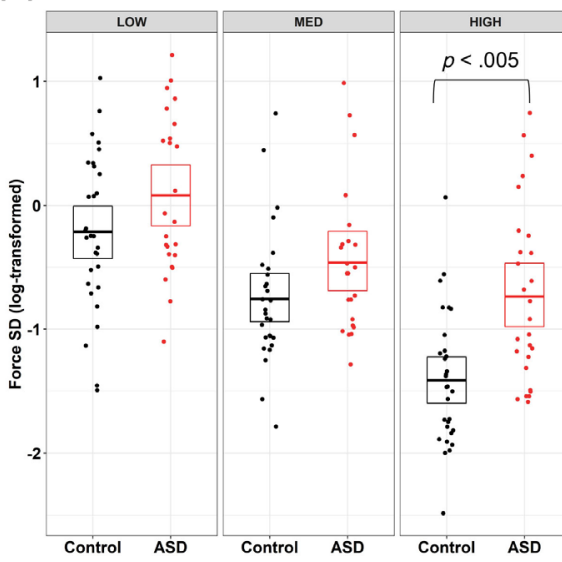

(c)

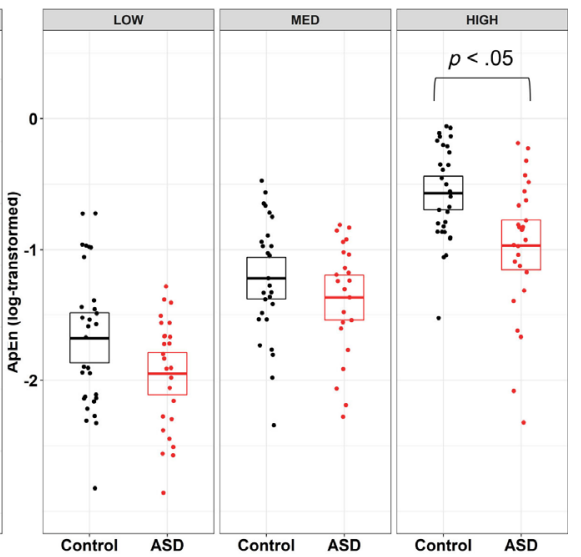

FIGURE 2 Results of the mixed effects models for behavioral force measures, controlling for age and sex. (a) Mean force was not different in individuals with ASD and TD controls, nor did it scale with gain. (b) Force SD significantly decreased with increasing gain (gain main effect), and was higher in individuals with ASD overall (Group main effect). There was also a significant interaction of gain and group, such that individuals with ASD showed greater force SD compared to TD controls at high gain, but not at low or medium gain. (c) Approximate Entropy (ApEn) significantly increased with increasing gain (gain main effect), and also was reduced in ASD relative to TD controls (Group main effect). No gain by group interaction was observed, though post-hoc group comparisons are presented for ease of comparison 
ApEn increased with increases in visual gain (Figure 2c; gain main effect: $\left.F_{(2,104.66)}=129.16, p<.001\right)$. Individuals with ASD showed reduced ApEn (i.e., reduced force entropy) compared to TD controls (Figure 2c; group main effect: $F_{(1,51.75)}=8.33, p<.01$ ), and the magnitude of this difference was similar across gain levels (group $\times$ gain interaction: $\left.F_{(2,104.75)}=1.45, p=.24\right)$. Increased age was associated with greater ApEn (age main effect: $F_{(1,51.87)}=9.53, p<.005$ ), and age-associated increases in ApEn were similar across groups (group $\times$ age interaction: $F_{(1,51.60)}=1.05, p=.31$ ).

\section{2 | Brain activation results}

Brain activation results are described as contralateral (i.e., left hemisphere) or ipsilateral (i.e., right hemisphere) relative to motor behavior with the right hand. In the visuomotor-rest contrast, BOLD activation of multiple regions of the visuomotor network scaled with increases in visual gain, including bilateral posterior parietal cortex (V5, SPL), bilateral primary (M1) and premotor cortex, bilateral cerebellar Crus I, bilateral middle cingulate cortex, contralateral (i.e., left, relative to the right hand used in the experiment) middle occipital gyrus, and ipsilateral (i.e., right) supramarginal gyrus. Figure S2 and Table S2 show brain regions with BOLD activation that scaled with visual gain during visuomotor behavior (gain main effect).

Five regions showed significant group $\times$ gain level effects (Figure 3; Table 2), including bilateral SMA, bilateral SPL, contralateral middle frontal gyrus (MFG), and contralateral inferior frontal gyrus (IFG). Except for contralateral IFG, brain activation in these regions scaled with gain level more strongly in individuals with ASD relative to TD controls resulting in increased activation in ASD compared to TD at high gain (Table S3). For contralateral IFG, this pattern of activation was similar, although the groups were not significantly different at high gain. Additionally, there was a significant group $\times$ age effect in bilateral medial visual cortex $\left(F_{(1,50)}=15.30, p<.005\right)$. Activation in bilateral V1 increased with age in ASD, but decreased with age in TD controls.

Brain activation results for visuomotor-vision and vision-rest contrasts are in Supporting Information Results 1 and 2, respectively.

\section{3 | Visuomotor-dependent functional connectivity results}

\subsection{1 | Functional connectivity differences between individuals with ASD and TD controls}

Visuomotor-dependent functional connectivity between ipsilateral IPL and a cluster in contralateral prefrontal cortex extending from IFG into ventral premotor cortex (contralateral PMv) differed between groups (Table 3; Figure 4). TD controls showed similar ipsilateral IPL-contralateral PMv connectivity during visuomotor action and rest, while individuals with ASD showed a visuomotor-

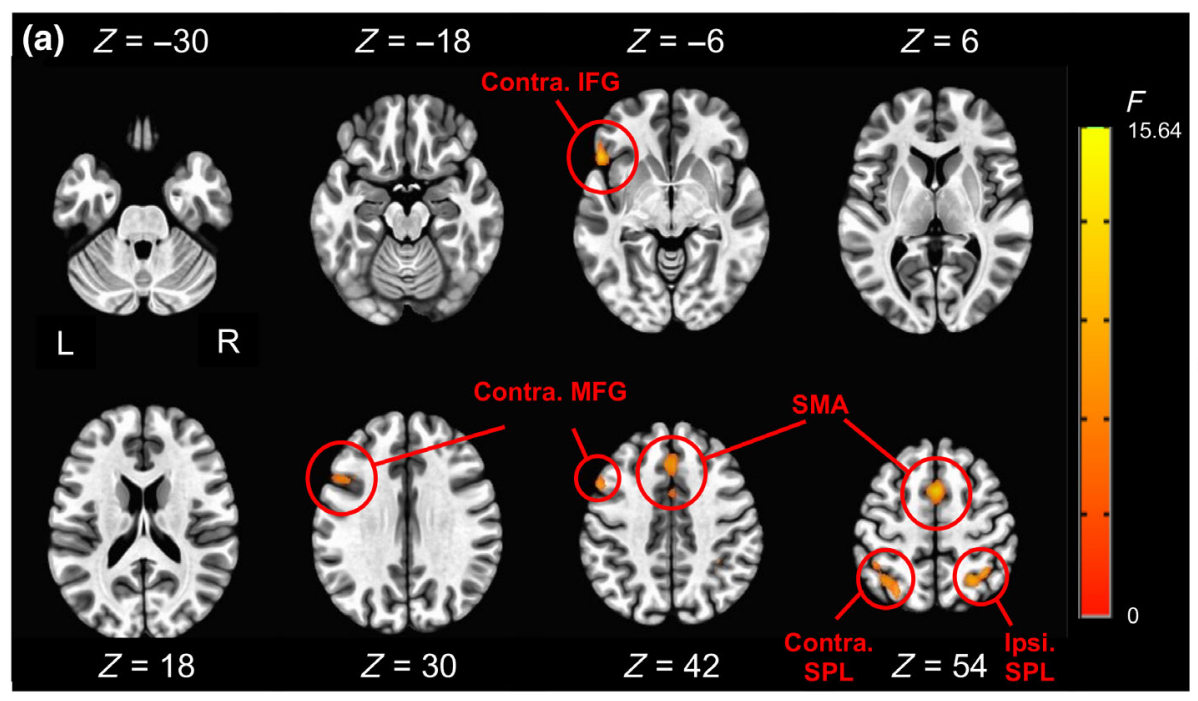

(b)

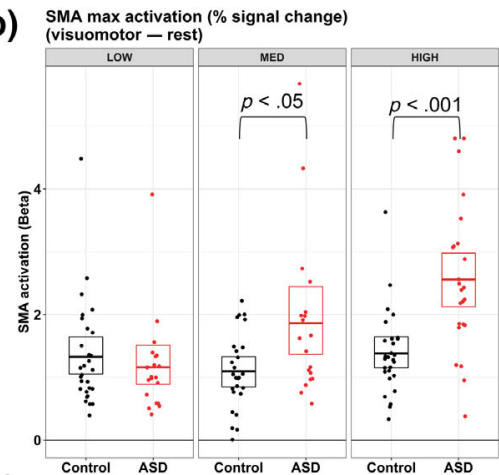

(c)

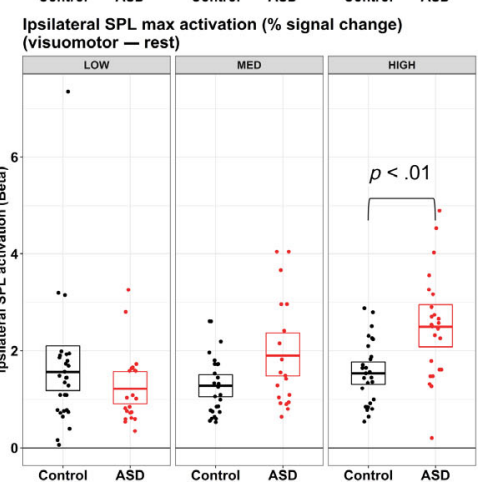

FIGURE 3 (a) Axial slices showing regions with activation that scaled with gain level for visuomotor-rest contrasts for individuals with ASD more than for TD controls. The color bar ranges from $F=0$ to $F=15.64$, with an activation threshold of $\alpha<.05$, corrected for multiple comparisons. (b) Individuals with ASD show increased SMA activation relative to TD that scaled in severity with increases in gain. (c) Ipsilateral (right) superior parietal lobule activation was greater in ASD compared to TD, especially during high gain 
TABLE 2 Brain regions showing significant group $\times$ visual gain interactions in activation in the linear mixed effects model (3dLME), controlling for age and sex

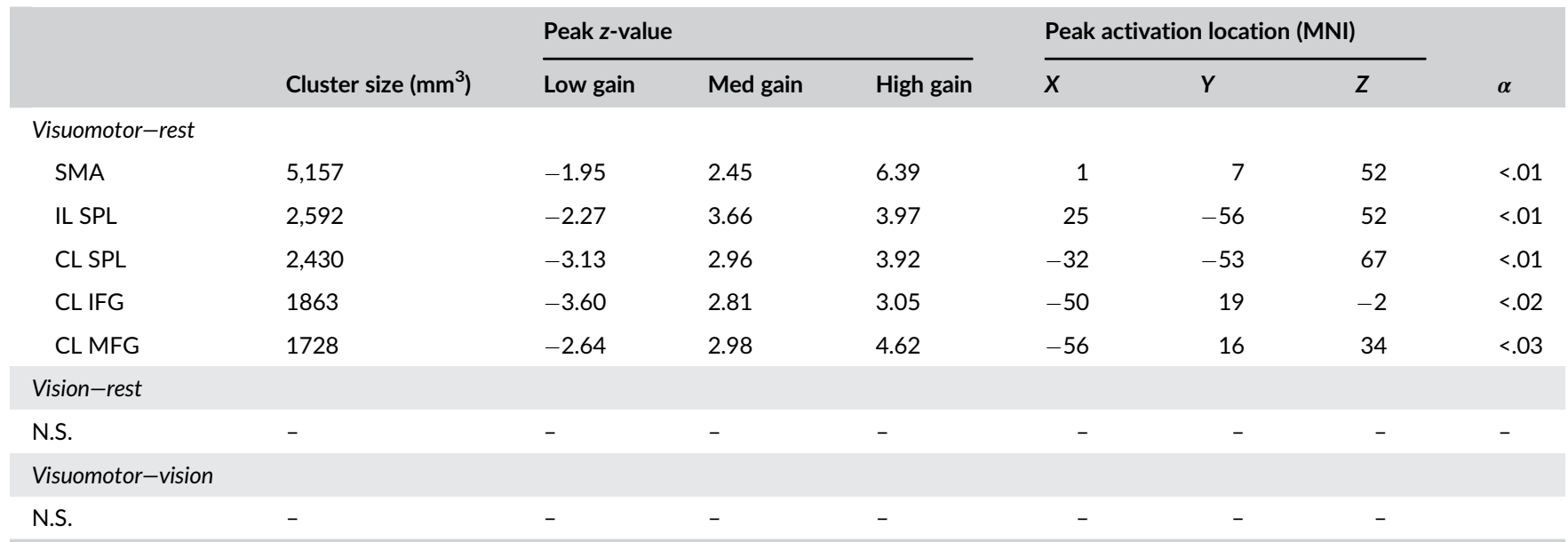

Note: Voxel-wise $p<.005 ; \alpha<.05$; clusters $\geq 57$ voxels $\left(1,539 \mathrm{~mm}^{3}\right)$ were retained; positive values reflect ASD > TD; peak activation location is in MNI (Montreal Neurologic Institute) coordinate space.

Abbreviations: CL, contralateral/left; IFG, inferior frontal gyrus; IL, ipsilateral/right; MFG, middle frontal gyrus; SMA, supplementary motor area; SPL, superior parietal lobule.

TAB LE 3 Brain regions showing significant group, gain, or group $\times$ age interactions in visuomotor-dependent functional connectivity in the linear mixed effects model (3dLME), controlling for age and sex

\begin{tabular}{|c|c|c|c|c|c|c|}
\hline & Cluster size $\left(\mathrm{mm}^{3}\right)$ & Peak F-value & \multicolumn{3}{|c|}{ Peak connectivity location (MNI) } & $\alpha$ \\
\hline \multicolumn{7}{|l|}{ Group (main effect) } \\
\hline IL IPL-CL putamen & 3,132 & 17.85 & -26 & 7 & 10 & $<.01$ \\
\hline IL IPL-CL PMv & 2,943 & 15.93 & -38 & 43 & 10 & $<.01$ \\
\hline \multicolumn{7}{|l|}{ Gain (main effect) } \\
\hline IL SPL-CL insula & 891 & 10.06 & -53 & 28 & -8 & $<.02$ \\
\hline IL SPL-CL IFG & 729 & 10.84 & -56 & 16 & 13 & $<.03$ \\
\hline CL SPL-CL IFG & 621 & 10.64 & -44 & 28 & 1 & $<.05$ \\
\hline IL V/VI-CL IFG & 837 & 13.37 & -47 & 25 & 4 & $<.02$ \\
\hline CL Crus I-ant cingulate & 5,805 & 20.90 & -5 & 43 & 13 & $<.01$ \\
\hline CL Crus I-Bilat V1 & 3,726 & 16.70 & 7 & -89 & -8 & $<.01$ \\
\hline CL Crus I-IL OFC & 3,186 & 25.46 & 13 & 58 & -11 & $<.01$ \\
\hline CL Crus I-CL IFG & 1998 & 16.76 & -29 & 19 & -23 & $<.02$ \\
\hline CL Crus I-mid cingulate & 1,620 & 14.00 & -11 & 25 & 31 & $<.04$ \\
\hline IL Crus I-ant cingulate & 3,834 & 21.28 & -8 & 40 & 13 & $<.01$ \\
\hline IL Crus I-Bilat OFC & 4,050 & 35.13 & 13 & 55 & -11 & $<.01$ \\
\hline IL V/VI-IL MFG/IFG & 2,511 & 19.32 & 10 & 61 & -8 & $<.01$ \\
\hline IL V/VI-IL Crus I/CL lingual & 2,133 & 23.56 & 10 & -74 & -20 & $<.02$ \\
\hline
\end{tabular}

Note: Voxel-wise $p<.005 ; \alpha<.05$; clusters $\geq 57$ voxels $\left(1,539 \mathrm{~mm}^{3}\right)$ were retained; Peak activation location is in MNI (Montreal Neurologic Institute) coordinate space.

Abbreviations: Ant, anterior; Bilat, bilateral; CL, contralateral/left; IFG, inferior frontal gyrus; IPL, inferior parietal lobule; IL, ipsilateral/right; Mid, middle; MFG, middle frontal gyrus; OFC, orbitofrontal cortex; PMv, ventral premotor cortex; SPL, superior parietal lobule; V/VI, cerebellar lobules V/VI. 

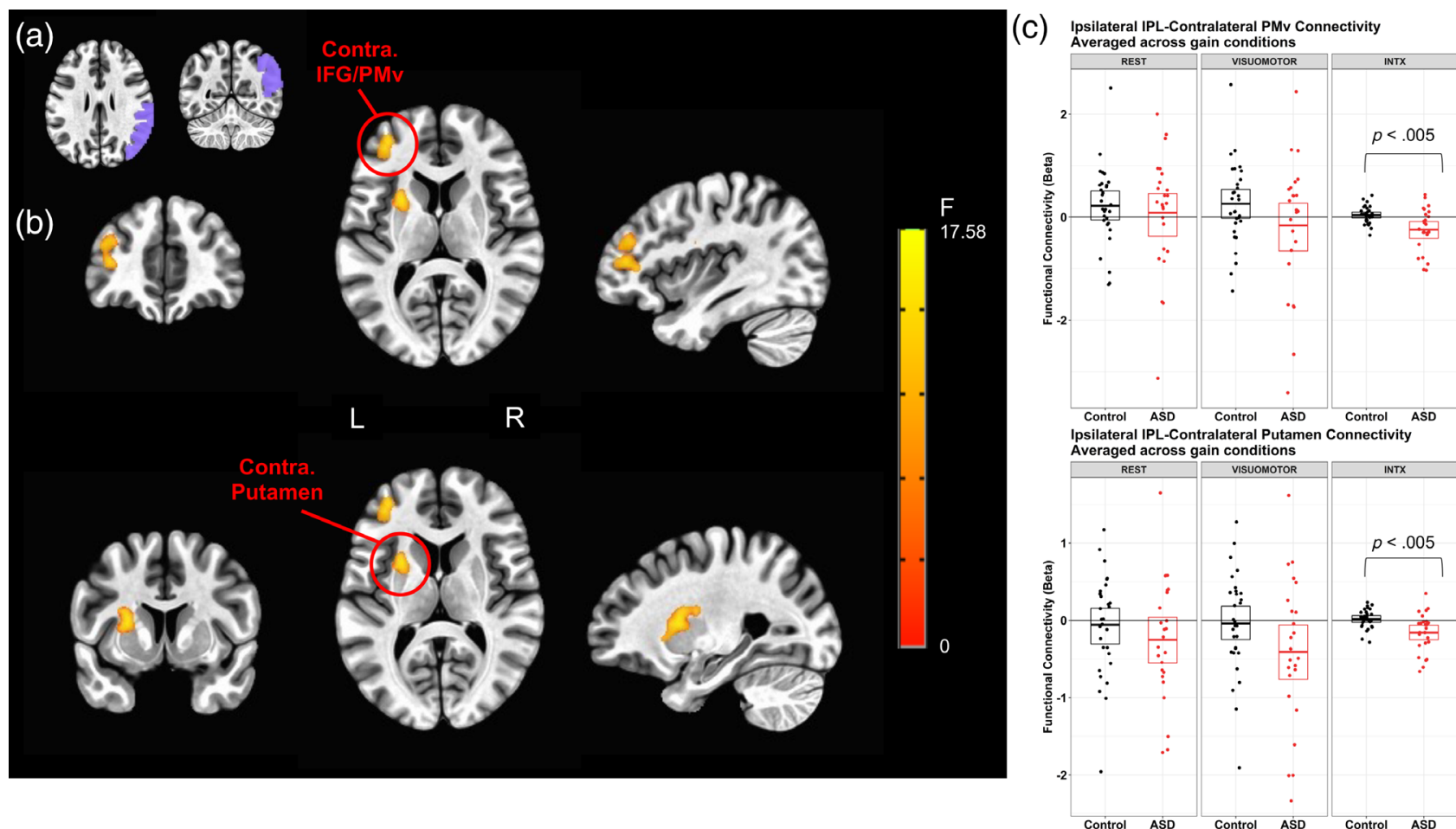

FIGURE 4 Results of the visuomotor-dependent connectivity analysis, corrected for age and sex. (a) The seed region used for the connectivity analysis-ipsilateral inferior parietal cortex (IPL). (b) Clusters in the contralateral ventral premotor cortex and putamen showing significantly reduced visuomotor-dependent connectivity with ipsilateral IPL in individuals with ASD compared to TD controls. The color bar ranges from $F=0$ to $F=17.53$, with an activation threshold of $\alpha<.05$, corrected for multiple comparisons. (c) The graphs display mean connectivity between the seed region and the cluster measured at rest and during visuomotor activity (VISUOMOTOR) for the two groups separately. The interaction (INTX) is the difference in connectivity for force minus rest, and is lower in ASD than TD controls in both clusters

dependent decrease in connectivity. Similarly, visuomotordependent functional connectivity between ipsilateral IPL and contralateral putamen was different between groups. TD controls showed similar ipsilateral IPL-contralateral putamen connectivity across visuomotor action and rest, while individuals with ASD showed reduced ipsilateral IPL-contralateral putamen connectivity during force compared to rest.

\subsection{2 | Functional connectivity differences across visual gain levels}

Visuomotor-dependent functional connectivity between ipsilateral SPL and several frontal regions varied by gain, including bilateral OFC, contralateral insula, and contralateral IFG (Table 3). Visuomotordependent connectivity between ipsilateral SPL and bilateral OFC as well as contralateral insula was stronger during medium and high gain relative to low gain. Visuomotor-dependent connectivity between ipsilateral SPL and contralateral IFG was stronger during high relative to low gain. Similarly, visuomotor-dependent functional connectivity between contralateral SPL and contralateral IFG was stronger during high relative to low gain.

Visuomotor-dependent functional connectivity between ipsilateral cerebellar lobules $\mathrm{V} / \mathrm{VI}$ and contralateral IFG varied by gain: visuomotor-dependent connectivity increased during high gain relative to low and medium gains (Table 3). Visuomotor-dependent functional connectivity between contralateral cerebellar lobules $\mathrm{V} / \mathrm{VI}$ and bilateral OFC was also greater during high and medium gain relative to low gain.

\subsection{3 | Age-associated group differences in functional connectivity}

Contralateral cerebellar Crus I and several regions showed significant group $\times$ age interactions in visuomotor-dependent functional connectivity, including bilateral caudate, bilateral anterior cingulate, bilateral V1, ipsilateral OFC, contralateral IFG, and bilateral middle cingulate. For bilateral caudate $\left(F_{(1,50)}=25.08, p<.001\right)$, bilateral anterior cingulate $\left(F_{(1,50)}=20.90, p<.001\right)$, and bilateral middle cingulate $\left(F_{(1,50)}\right.$ $=14.00, p<.005)$, connectivity with contralateral Crus I during visuomotor action increased with age in individuals with ASD but remained stable across age in TD controls, resulting in increased visuomotor-dependent connectivity differences with age in ASD relative to TD controls. For contralateral IFG $\left(F_{(1,50)}=16.76, p<.005\right)$, ipsilateral OFC $\left(F_{(1,50)}=25.46, p<.001\right)$, and bilateral V1 $\left(F_{(1,50)}\right.$ $=16.70, p<.005)$, connectivity with contralateral Crus I during visuomotor action increased with age in both groups but showed 
larger increases in individuals with ASD, resulting in increased visuomotor-dependent connectivity differences with age in ASD relative to TD controls (Figure 5a).

Ipsilateral cerebellar Crus I and two prefrontal cortical regions showed significant group $\times$ age interactions in visuomotor-dependent functional connectivity, including bilateral anterior cingulate and bilateral OFC. For bilateral anterior cingulate $\left(F_{(1,50)}=21.28, p<.005\right)$ and bilateral OFC $\left(F_{(1,50)}=35.13, p<.001\right)$, connectivity with ipsilateral Crus I increased with age more sharply in ASD than in TD controls (Figure 5b).

Ipsilateral cerebellar lobules V/VI and two regions showed significant group $\times$ age interactions in visuomotor-dependent functional connectivity, including ipsilateral middle and frontal gyri and a cluster spanning ipsilateral medial cerebellum lobule $\mathrm{V}$ and contralateral lingual gyrus. For ipsilateral middle and frontal gyri $\left(F_{(1,50)}=19.32\right.$, $p<.005)$, both groups showed increased connectivity with ipsilateral lobules V/VI during visuomotor action with age, though this increase was larger in individuals with ASD. For ipsilateral lobule Vcontralateral lingual gyrus $\left(F_{(1,50)}=23.56, p<.005\right)$, connectivity with ipsilateral lobules $\mathrm{V} / \mathrm{VI}$ during rest increased with age in individuals with ASD but showed larger increases with age in TD controls, while both groups showed similar increases in connectivity during visuomotor action with age. This resulted in increased visuomotor- dependent connectivity differences with age in ASD relative to TD controls (Figure $5 \mathrm{c}$ ). Examination of the scatter plots revealed potential outliers for individuals $>30$ years that may have been driving correlations. As a conservative approach, we conducted the analyses again excluding those individuals (two ASD, two TD). Correlations that remain significant without these individuals are marked in Figure 5, and full results for these analyses are included in Supporting Information Results 3, Figure S4.

\section{4 | Relationships between visuomotor behavior, brain function, and ASD symptoms}

There were no significant relationships between force SD and ApEn with ASD symptom severity, as measured by either the ADOS or the ADI (Table S4).

Across participants and gain levels, increased contralateral SPL activation during force was associated with reduced ApEn $\left(F_{(1,131.55)}\right.$ $=6.48, p=.01)$ and increased force $S D\left(F_{(1,135.54)}=14.08, p<.001\right)$. For individuals with ASD, reduced bilateral SMA activation during force was associated with increased severity of ADI-rated social $\left(F_{(1,11.87)}=10.83, p<.001\right)$ and communication abnormalities $\left(F_{(1,13.31)}=7.31, p=.02\right)$. (a)

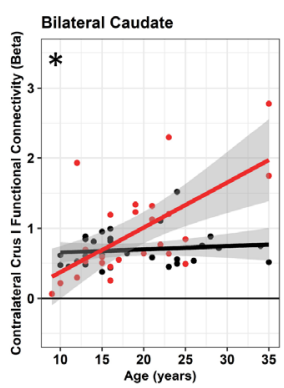

Contralateral Crus I
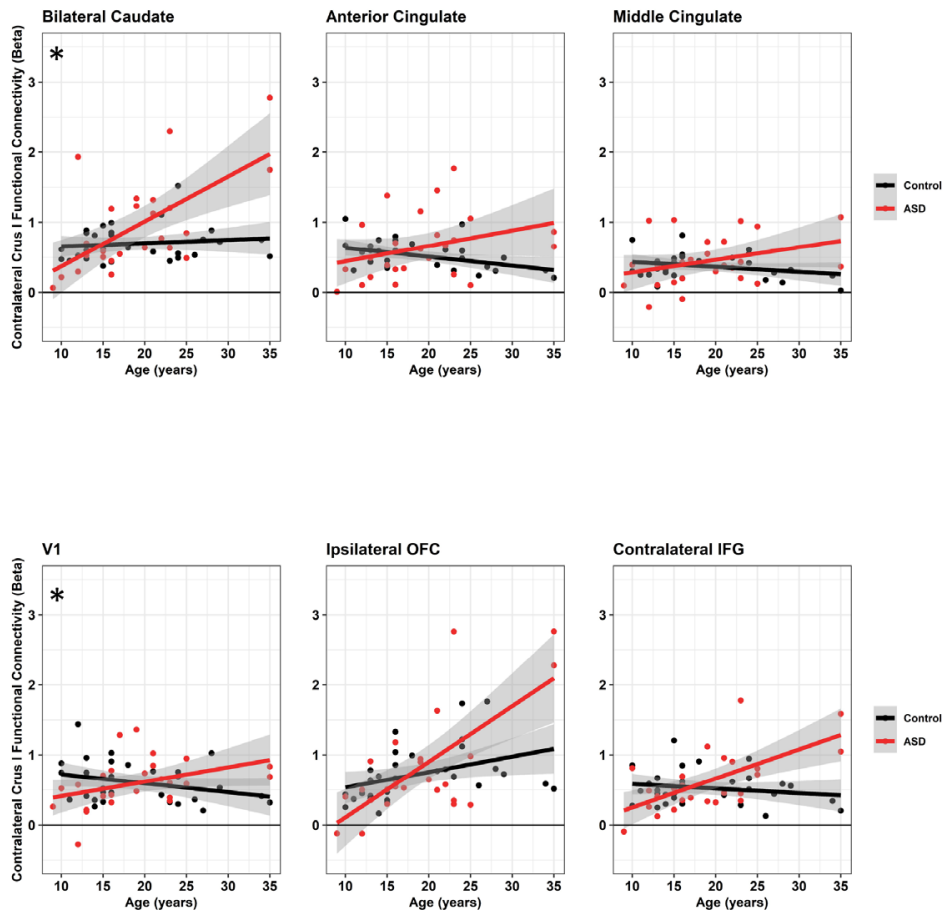

(c)

(b)

\section{Ipsilateral Crus I}
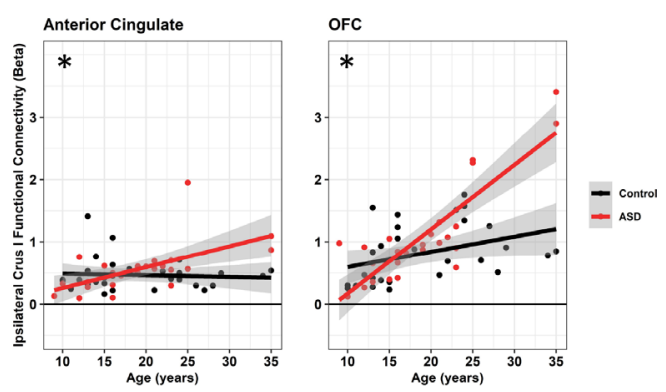

(c) Ipsilateral Lobules V/VI
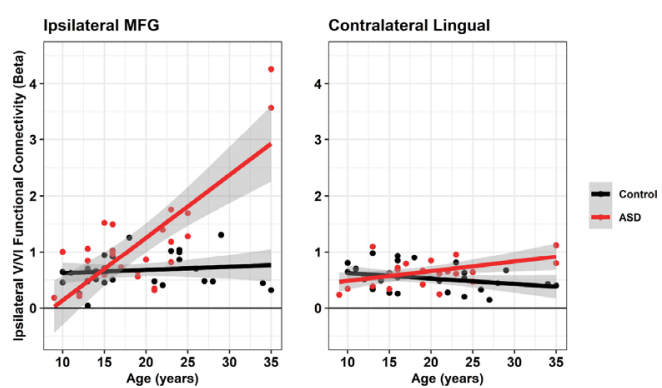

FIG URE 5 Age-related group differences in visuomotor-dependent connectivity. Scatterplots display the difference in connectivity between the two indicated brain regions during visuomotor activity versus rest (visuomotor-rest) for each participant. Red dots represent ASD participants; black dots represent TD control participants. Solid lines represent linear trend lines for age for each group, and shaded areas indicate $95 \%$ confidence intervals. Across most regions, ASD participants have reduced visuomotor-dependent connectivity at younger ages compared to TD control participants. Asterisks $\left(^{*}\right)$ in the upper-left portion of graphs denote group $\times$ age interactions which remained significant after a second analysis excluding the four individuals in our data set $>30$ years (two ASD; two TD) 
Increased visuomotor-dependent functional connectivity between contralateral Crus I and bilateral V1 was associated with reduced force $S D\left(F_{(1,130.45)}=7.14, p<.01\right)$. Increased connectivity between ipsilateral lobules $\mathrm{V} / \mathrm{VI}$ and ipsilateral MFG-IFG was associated with increased ApEn $\left(F_{(1,116.88)}=7.67, p<.01\right)$. The relationship between force $S D$ and connectivity between ipsilateral Crus I and bilateral anterior cingulate differed between groups (group $\times$ force $\left.S D ; F_{(1,130.93)}=11.94, p<.001\right)$; increased connectivity was associated with reduced force SD in TD controls but not in individuals with ASD. Increased ADOS-rated ASD symptom severity was associated with reduced connectivity between contralateral Crus I and contralateral IFG $\left(F_{(1,22)}=7.92, p=.01\right)$, and reduced connectivity between ipsilateral lobules $\mathrm{V} / \mathrm{VI}$ and ipsilateral MFG-IFG $\left(F_{(1,22)}\right.$ $=7.15, p=.01)$

\section{DISCUSSION}

We provide new evidence of atypical brain activation and functional connectivity associated with precision visuomotor impairments in ASD. Five key findings are highlighted. First, we show that increases in force variability are more severe at high relative to low visual gain suggesting impaired ability to process increased visual feedback. Second, we document that individuals with ASD show increased activation in bilateral SMA, bilateral SPL, and contralateral MFG/IFG during visuomotor behavior. These differences were more pronounced at high visual gain suggesting reduced modulation of local circuit activity within sensory processing and motor control regions during precision behavior. Third, we report reduced visuomotor-dependent functional connectivity between ipsilateral IPL and contralateral PMv as well as ipsilateral IPL and contralateral putamen in ASD, implicating diminished integration of parietal feedback with cortical and subcortical motor planning systems. Fourth, we observe that individuals with ASD show age-associated increases in functional connectivity of cerebellum and cerebral cortex, including occipital, medial prefrontal, and lateral prefrontal cortices, suggesting delayed cerebellar-cortical maturation in ASD. Last, we find that reduced cerebellar-prefrontal connectivity is associated with reduced force entropy and increased ASD symptoms, suggesting that atypical development of cerebellar-cortica networks may underpin sensorimotor and core social-communicative impairments.

\section{1 | Visuomotor behavior in ASD}

Consistent with our prior studies (Mosconi et al., 2015; Wang et al., 2015), we found that visually guided precision force variability is elevated in ASD, especially when visual feedback is amplified, suggesting that reduced visuomotor precision in ASD involves difficulty processing increased visual feedback information. Individuals with ASD also showed reduced entropy compared to TD controls implicating deficits integrating feedforward and multiple sensory feedback processes operating on different time scales.
Our results implicating deficient sensory feedback processing in ASD are consistent with prior studies demonstrating that patients may show atypical biases towards select sensory inputs, such as proprioceptive feedback, rather than integrating feedback across multiple sensory modalities (Haswell et al., 2009; Izawa et al., 2012). Results from the present study add evidence that sensorimotor impairment in ASD involves a reduced ability to integrate multisensory feedback to precisely and dynamically modulate behavior.

\section{2 | Visuomotor brain function in ASD}

Activation across multiple brain regions scaled with increases in visual gain during visuomotor action, consistent with prior studies suggesting that the ability to reactively adjust ongoing sensorimotor behavior is dependent on scaling of activation across widely distributed cortical and cerebellar neural circuits (Vaillancourt, Mayka, \& Corcos, 2006) involving posterior parietal and temporo-parietal cortices, motor cortex (Glickstein \& Stein, 1991), cerebellum, thalamo-M1 circuits (Calhoun et al., 2001), and striatal nuclei (Bostan, Dum, \& Strick, 2018). Individuals with ASD demonstrated atypical scaling of visuomotor network activation across visual gains relative to controls, consistent with results observed in their behavioral data. This novel finding indicates a failure to modulate performance in response to changes in sensory feedback quality. Our finding relating SPL activation with increased force variability and reduced entropy provides additional evidence that visuomotor impairments in ASD reflect deficits in cortical processing of multi-sensory feedback information. Together, these findings indicate that local activations of motor planning (SMA and MFG) and spatial processing (SPL) circuits show greater reactivity to amplifications of visual feedback that are associated with less precise visuomotor behavior in ASD.

\section{3 | Visuomotor-dependent functional connectivity in ASD}

IPL-PMv network functional connectivity supports the planning and precision of visually guided grasping (Bonini et al., 2010) while also serving to modify outgoing motor commands in response to sensory feedback (Igelstrom \& Graziano, 2017; Schnell et al., 2007). Reductions in the functional connectivity of this pathway during visuomotor control in ASD indicate a selective decoupling of multisensory parietal input and motor planning circuits during behavior. These results suggest local sensory feedback processing and motor control circuits may function more independently in ASD than in TD, with less integration between parietal and frontal regions (Just, Cherkassky, Keller, Kana, \& Minshew, 2007; Kana, Keller, Cherkassky, Minshew, \& Just, 2009), and rely on local circuit modulation as reflected by increased activation of SMA, MFG, and SPL in the present study.

Our finding of reduced functional connectivity between ipsilateral IPL and contralateral putamen in ASD implicates abnormal integration of visual feedback and motor timing and amplitude mechanisms during 
sensory guided motor refinement (Prodoehl et al., 2008). Consistent with our findings, multiple studies have documented striatal dysfunction in ASD, including increased activation during precision grip force control (Unruh et al., 2019) and reduced functional connectivity with V1 during finger tapping (Villalobos, Mizuno, Dahl, Kemmotsu, \& Muller, 2005). Our results extend these findings to indicate that precision grip force impairments in ASD involve decreased functional connectivity between posterior parietal cortical circuits involved in integrating sensory feedback information and striatal mechanisms involved in modulating the timing and amplitude of action output.

Reduced functional connectivity between ipsilateral IPL and both contralateral PMv and putamen in ASD may implicate reduced lateralization of sensorimotor circuits involved in precisely adjusting precision manual behavior. Parietal multisensory processing is right lateralized in TD (Sack et al., 2002; Tsakiris, Longo, \& Haggard, 2010), while unilateral movements are planned, executed, and modulated through contralateral premotor-subcortical functional networks, including contralateral PMv and putamen (Pool, Rehme, Fink, Eickhoff, \& Grefkes, 2014). This would be consistent with previous studies documenting reduced leftward and increased rightward resting state functional connectivity of sensorimotor networks in children and adolescents with ASD (Cardinale, Shih, Fishman, Ford, \& Muller, 2013; Floris et al., 2016). Alternatively, reduced ipsilateral IPL-contralateral PMv functional connectivity may reflect broader reductions in parietal-premotor functional connectivity, independent of effector side. As we only tested unimanual (right) precision motor performance in a predominantly right-handed sample, left PMv was likely disproportionately integrated with IPL relative to right PMv. Reduced functional connectivity between ipsilateral IPL and ipsilateral PMv may be more readily detectable in studies of left hand behavior. Studies of motor behavior across both hands will clarify whether reduced functional connectivity observed in the present study reflects reduced functional lateralization or more broadly diminished parietalpremotor functional connectivity.

\subsection{Age-associated increases in cerebellar- cortical functional connectivity in ASD}

Visuomotor-dependent cerebellar-striatal (caudate) and cerebellarcortical (ACC, OFC, IFG, MFG, V1, Lingual) functional connectivity showed stronger age-associated increases in ASD relative to TD controls. One possible explanation for this finding is that the development of cerebellar processes involved in the modulation of striatal and prefrontal circuits is delayed in ASD but catches up to (or even surpasses) neurotypical levels in adulthood (Yim, Aertsen, \& Kumar, 2011). Although the current cross-sectional study examined largely older adolescents and adults with ASD, these results are consistent with prior studies showing atypical timing of motor output in children with ASD (D'Cruz et al., 2009; Wang et al., 2015), and associations between striatal volumes and repetitive behaviors that implicate deficits in efficient selection and assembly of motor programs (Langen et al., 2014). Age-associated increases in functional connectivity also may reflect greater consolidation of cerebellar-striatal and cerebellarcortical networks, suggesting reduced flexibility of sensorimotor network selection and increased reliance on select networks in ASD. Reduced network flexibility may be detrimental to higher-order, complex cognitive processes also supported by these networks, as has been previously suggested (Khan et al., 2015; Minshew, Sweeney, \& Luna, 2002). It also is possible that these findings reflect biases in our sample based on the potential for adults with ASD who volunteer for fMRI studies being less severely affected than children with ASD who may be supported in their involvement by their parents or caregivers.

Atypical age-associations of cerebellar Crus I-IFG functional connectivity in ASD implicate maturational processes involved in reactively adjusting outgoing motor commands during precision behavior. Findings that functional connectivity of cerebellum and both bilateral ACC and contralateral OFC are reduced in ASD suggests delayed development of networks involved in flexibly modifying force output during goal-directed behavior, consistent with studies from our lab (Mosconi et al., 2015; Wang et al., 2015) and others (Lidstone et al., 2020; Morimoto, Hida, Shima, \& Okamura, 2018) showing increased motor variability and reduced precision motor accuracy in ASD, especially in younger children. These precision sensorimotor tests may also be more challenging to individuals with ASD than TD based on studies showing that lateral cerebellum and prefrontal cortical circuits, including ACC and OFC, show greater coactivation during more challenging task or cognitive conditions (Braver et al., 1997; Rypma, Prabhakaran, Desmond, Glover, \& Gabrieli, 1999; Stoodley \& Schmahmann, 2009).

Age-associated group differences were also observed in Crus Ivisual cortex functional connectivity. Visual input to cerebellum via pontine nuclei is used to guide adjustments of the motor command relayed to motor cortex (Glickstein, 2000). Atypical development of this network implicates abnormal processing of visual feedback information that may contribute to increased variability and reduced entropy of sustained motor actions. Consistent with these findings, reduced cerebellarcortical functional connectivity during rest (Ramos, Balardin, Sato, \& Fujita, 2018; Wang et al., 2019) and motor action (Mostofsky et al., 2009) has been reported in individuals with ASD, though increased cerebellar-cortical functional connectivity during rest also has been indicated when age is included as a covariate (Khan et al., 2015).

\subsection{Brain network function and behavior}

Cerebellar-prefrontal brain alterations in circuits supporting visuomotor behavior and higher-order language and socioemotional processing were related to visuomotor impairments and more severe core symptoms in ASD (Stoodley \& Schmahmann, 2009). Increased cerebellar-V1 and cerebellar-MFG-IFG functional connectivity was associated with lower motor variability suggesting that reorganization of these network functions may compensate for deficits in visuomotor network connectivity and local circuit modulation. Cerebellar-anterior cingulate connectivity was associated with reduced force variability in TD controls but not in individuals with ASD. Relatedly, cerebellaranterior cingulate connectivity increased with age in individuals with 
ASD only. Anterior cingulate facilitates movement onset and aids in action selection and error detection (Paus, 2001). Prior studies have identified increased co-activation of anterior cingulate and cerebellum during finger tapping (Lench, DeVries, \& Hanlon, 2017). These findings together suggest that, with age, individuals with ASD may show increased cerebellar-anterior cingulate functional connectivity during visuomotor behavior, but that greater integration of this pathway does not appear to contribute to increased precision of visuomotor output in patients. The relationship of cerebellar-prefrontal functional connectivity to reduced force entropy and more severe socialcommunication abnormalities suggests that neurodevelopmental disruptions of these circuits may impact both early maturing sensorimotor behaviors and more complex social-communication abilities.

\section{6 | Limitations}

The present results should be considered in the context of several limitations. First, while task-based fMRI provides important advantages for tracking brain-behavior relationships relative to resting-state fMRI (Greene et al., 2018), this method presents limitations for assessing younger and more severely impaired individuals, including those with comorbid intellectual or developmental disabilities. While these methods may be less scalable across the ASD population relative to other imaging approaches (e.g., resting state fMRI, EEG/ERP), they do have the benefit of being a direct test of the visuomotor system in action. Our results identify multiple subregions and functional pathways in the visuomotor system that now can be probed at younger ages and across the range of severity using less powerful but more scalable strategies. Second, our findings of age-dependent group differences in both task activation and functional connectivity highlight the need for longitudinal testing to characterize mechanisms of atypical age relationships and to determine primary and compensatory neurodevelopmental processes associated with sensorimotor and core deficits of ASD. Third, our sample of participants with ASD and controls differed on verbal IQ. Performance on the motor task we employed does not rely on verbal ability, and we did not see associations between verbal ability and sensorimotor behavior in this study or our prior studies (Mosconi et al., 2015; Wang et al., 2015. Differences in verbal IQ would therefore not be expected to strongly influence the findings. Finally, our sample was predominately composed of biological males, and there were proportionately more males in our group with ASD relative to controls, consistent with population rates. Although we did not see evidence of sex effects or group by sex interactions for precision sensorimotor behavior or brain function in this study or our previous work (Unruh et al., 2021), future studies should investigate whether underlying brain processes are similarly affected across all sexes and genders.

\section{7 | Conclusions}

We show that atypical sensorimotor behavior in ASD is linked to increased functional activation and decreased task-dependent functional connectivity throughout the visuomotor network, including posterior parietal, frontal cortical, striatal, and cerebellar regions, and that these dysfunctions vary according to the quality of sensory feedback. Combined with our findings that brain alterations are associated with visuomotor impairment and core ASD symptoms, our results suggest that atypical modulation of sensory feedback information during precision refinement of ongoing motor behavior contributes to multiple developmental disruptions in affected individuals.

\section{ACKNOWLEDGMENTS}

This work was supported by the National Institutes of Health (grant numbers P50 ACE HD055751 (to J.A.S.), R01 MH112734 (to M.M.), K23 MH092696 (to M.M.), R21 AG065621 (to Z.W.), University of Florida CTSI Pilot Award UL1TR001427 (to Z.W.), U54 HD090216; and a Kansas Center for Autism Research and Training (K-CART) Research Investment Council Strategic Initiative Grant (to M.M.).

\section{CONFLICT OF INTEREST}

Drs. Stephen Coombes and David Vaillancourt are co-founders and managers of Neuroimaging Solutions, LLC. The other authors have no competing interests to declare.

\section{AUTHOR CONTRIBUTIONS}

Rebecca J. Lepping: Data curation; formal analysis; writing-original draft. Walker S. McKinney: Data curation; formal analysis; writingoriginal draft. Grant C. Magnon: Writing-review \& editing. Sarah K. Keedy: Investigation; writing-review \& editing. Zheng Wang: Data curation; investigation; software; writing-review \& editing. Stephen A. Coombes: Methodology; software; writing-review \& editing. David E. Vaillancourt: Conceptualization; methodology; software; writing-review \& editing. John A. Sweeney: Conceptualization; methodology; funding acquisition; Writing-review \& editing. Matthew W. Mosconi: Conceptualization; funding acquisition; supervision; writing-review \& editing.

We gratefully acknowledge the families who participated in these studies.

\section{DATA AVAILABILITY STATEMENT}

The data that support the findings of this study are available from the corresponding author upon reasonable request.

\section{ORCID}

Rebecca J. Lepping (iD) https://orcid.org/0000-0001-9269-051X Walker S. McKinney (D) https://orcid.org/0000-0001-5281-3749 David E. Vaillancourt (D) https://orcid.org/0000-0002-5663-6476

\section{REFERENCES}

Allen, G., \& Courchesne, E. (2003). Differential effects of developmental cerebellar abnormality on cognitive and motor functions in the cerebellum: An fMRI study of autism. The American Journal of Psychiatry, 160(2), 262-273. https://doi.org/10.1176/appi.ajp.160.2.262

Allen, G., Muller, R. A., \& Courchesne, E. (2004). Cerebellar function in autism: Functional magnetic resonance image activation during a simple motor task. Biological Psychiatry, 56(4), 269-278. https://doi.org/ 10.1016/j.biopsych.2004.06.005 
Amemiya, K., Morita, T., Saito, D. N., Ban, M., Shimada, K., Okamoto, Y., ... Naito, E. (2019). Local-to-distant development of the cerebrocerebellar sensorimotor network in the typically developing human brain: A functional and diffusion MRI study. Brain Structure \& Function, 224(3), 1359-1375. https://doi.org/10.1007/s00429-018-01821-5

American Psychiatric Association. (2000). Diagnostic and statistical manual of mental disorders (DSM-IV-TR). Washington, DC: American Psychiatric Association.

American Psychiatric Association. (2013). Diagnostic and statistical manual of mental disorders (DSM-5). Washington, DC: American Psychiatric Association.

Bal, V. H., Fok, M., Lord, C., Smith, I. M., Mirenda, P., Szatmari, P., ... Zaidman-Zait, A. (2020). Predictors of longer-term development of expressive language in two independent longitudinal cohorts of language-delayed preschoolers with autism spectrum disorder. Journal of Child Psychology and Psychiatry, 61(7), 826-835. https://doi.org/10. 1111/jcpp.13117

Bojanek, E. K., Wang, Z., White, S. P., \& Mosconi, M. W. (2020). Postural control processes during standing and step initiation in autism spectrum disorder. Journal of Neurodevelopmental Disorders, 12(1), 1. https://doi.org/10.1186/s11689-019-9305-x

Bonini, L., Rozzi, S., Serventi, F. U., Simone, L., Ferrari, P. F., \& Fogassi, L. (2010). Ventral premotor and inferior parietal cortices make distinct contribution to action organization and intention understanding. Cerebral Cortex, 20(6), 1372-1385. https://doi.org/10.1093/cercor/ bhp200

Bostan, A. C., Dum, R. P., \& Strick, P. L. (2018). Functional anatomy of basal ganglia circuits with the cerebral cortex and the cerebellum. Progress in Neurological Surgery, 33, 50-61. https://doi.org/10.1159/ 000480748

Braver, T. S., Cohen, J. D., Nystrom, L. E., Jonides, J., Smith, E. E., \& Noll, D. C. (1997). A parametric study of prefrontal cortex involvement in human working memory. Neurolmage, 5(1), 49-62. https://doi.org/ 10.1006/nimg.1996.0247

Calhoun, V. D., Adali, T., McGinty, V. B., Pekar, J. J., Watson, T. D., \& Pearlson, G. D. (2001). fMRI activation in a visual-perception task: Network of areas detected using the general linear model and independent components analysis. Neurolmage, 14(5), 1080-1088. https://doi.org/10.1006/nimg.2001.0921

Caminiti, R., Ferraina, S., \& Johnson, P. B. (1996). The sources of visual information to the primate frontal lobe: A novel role for the superior parietal lobule. Cerebral Cortex, 6(3), 319-328. https://doi.org/10. 1093/cercor/6.3.319

Cardinale, R. C., Shih, P., Fishman, I., Ford, L. M., \& Muller, R. A. (2013). Pervasive rightward asymmetry shifts of functional networks in autism spectrum disorder. JAMA Psychiatry, 70(9), 975-982. https://doi.org/ 10.1001/jamapsychiatry.2013.382

Chen, G., Saad, Z. S., Britton, J. C., Pine, D. S., \& Cox, R. W. (2013). Linear mixed-effects modeling approach to FMRI group analysis. Neurolmage, 73, 176-190. https://doi.org/10.1016/j.neuroimage.2013.01.047

Cisler, J. M., Bush, K., \& Steele, J. S. (2014). A comparison of statistical methods for detecting context-modulated functional connectivity in fMRI. Neurolmage, 84, 1042-1052. https://doi.org/10.1016/j. neuroimage.2013.09.018

Coll, S.-M., Foster, N. E. V., Meilleur, A., Brambati, S. M., \& Hyde, K. L. (2020). Sensorimotor skills in autism spectrum disorder: A meta-analysis. Research in Autism Spectrum Disorders, 76, 101570. https://doi. org/10.1016/j.rasd.2020.101570

Coombes, S. A., Corcos, D. M., Sprute, L., \& Vaillancourt, D. E. (2010). Selective regions of the visuomotor system are related to gain-induced changes in force error. Journal of Neurophysiology, 103(4), 2114-2123. https://doi.org/10.1152/jn.00920.2009

Cox, R. W. (1996). AFNI: Software for analysis and visualization of functional magnetic resonance neuroimages. Computers and Biomedical Research, 29(3), 162-173.
D'Cruz, A. M., Mosconi, M. W., Steele, S., Rubin, L. H., Luna, B., Minshew, N., \& Sweeney, J. A. (2009). Lateralized response timing deficits in autism. Biological Psychiatry, 66(4), 393-397. https://doi.org/ 10.1016/j.biopsych.2009.01.008

Diedrichsen, J. (2006). A spatially unbiased atlas template of the human cerebellum. Neurolmage, 33(1), 127-138. https://doi.org/10.1016/j. neuroimage.2006.05.056

Elliott, C., Vijayakumar, V., Zink, W., \& Hansen, R. (2007). National Instruments LabVIEW: A programming environment for laboratory automation and measurement. JALA: Journal of the Association for Laboratory Automation, 12(1), 17-24. https://doi.org/10.1016/j.jala.2006.07.012

Fan, L., Li, H., Zhuo, J., Zhang, Y., Wang, J., Chen, L., ... Jiang, T. (2016). The human brainnetome atlas: A new brain atlas based on connectional architecture. Cerebral Cortex, 26(8), 3508-3526. https://doi.org/10. 1093/cercor/bhw157

Floris, D. L., Barber, A. D., Nebel, M. B., Martinelli, M., Lai, M. C., Crocetti, D., ... Mostofsky, S. H. (2016). Atypical lateralization of motor circuit functional connectivity in children with autism is associated with motor deficits. Molecular Autism, 7, 35. https://doi.org/10.1186/ s13229-016-0096-6

Fonov, V., Evans, A. C., Botteron, K., Almli, C. R., McKinstry, R. C., Collins, D. L., \& Brain Development Cooperative, G. (2011). Unbiased average age-appropriate atlases for pediatric studies. Neurolmage, 54(1), 313-327. https://doi.org/10.1016/j.neuroimage.2010.07.033

Glickstein, M. (2000). How are visual areas of the brain connected to motor areas for the sensory guidance of movement? Trends in Neurosciences, 23(12), 613-617.

Glickstein, M., \& Stein, J. (1991). Paradoxical movement in Parkinson's disease. Trends in Neurosciences, 14(11), 480-482.

Glover, S., Wall, M. B., \& Smith, A. T. (2012). Distinct cortical networks support the planning and online control of reaching-to-grasp in humans. The European Journal of Neuroscience, 35(6), 909-915. https://doi.org/10.1111/j.1460-9568.2012.08018.x

Greene, A. S., Gao, S., Scheinost, D., \& Constable, R. T. (2018). Taskinduced brain state manipulation improves prediction of individual traits. Nature Communications, 9(1), 2807. https://doi.org/10.1038/ s41467-018-04920-3

Haswell, C. C., Izawa, J., Dowell, L. R., Mostofsky, S. H., \& Shadmehr, R. (2009). Representation of internal models of action in the autistic brain. Nature Neuroscience, 12(8), 970-972. https://doi.org/10.1038/ nn. 2356

Holiga, S., Hipp, J. F., Chatham, C. H., Garces, P., Spooren, W., D'Ardhuy, X. L., ... Dukart, J. (2019). Patients with autism spectrum disorders display reproducible functional connectivity alterations. Science Translational Medicine, 11(481), eaat9223. https://doi.org/10.1126/ scitranslmed.aat9223

Igelstrom, K. M., \& Graziano, M. S. A. (2017). The inferior parietal lobule and temporoparietal junction: A network perspective. Neuropsychologia, 105, 70-83. https://doi.org/10.1016/j. neuropsychologia.2017.01.001

Izawa, J., Pekny, S. E., Marko, M. K., Haswell, C. C., Shadmehr, R., \& Mostofsky, S. H. (2012). Motor learning relies on integrated sensory inputs in ADHD, but over-selectively on proprioception in autism spectrum conditions. Autism Research, 5(2), 124-136. https://doi.org/ 10.1002/aur.1222

Johnson, B. P., Rinehart, N. J., White, O., Millist, L., \& Fielding, J. (2013). Saccade adaptation in autism and Asperger's disorder. Neuroscience, 243, 76-87. https://doi.org/10.1016/j.neuroscience.2013.03.051

Just, M. A., Cherkassky, V. L., Keller, T. A., Kana, R. K., \& Minshew, N. J. (2007). Functional and anatomical cortical underconnectivity in autism: Evidence from an FMRI study of an executive function task and corpus callosum morphometry. Cerebral Cortex, 17(4), 951-961. https:// doi.org/10.1093/cercor/bhl006

Kana, R. K., Keller, T. A., Cherkassky, V. L., Minshew, N. J., \& Just, M. A. (2009). Atypical frontal-posterior synchronization of theory of mind 
regions in autism during mental state attribution. Social Neuroscience, 4(2), 135-152. https://doi.org/10.1080/17470910802198510

Khan, A. J., Nair, A., Keown, C. L., Datko, M. C., Lincoln, A. J., \& Muller, R. A. (2015). Cerebro-cerebellar resting-state functional connectivity in children and adolescents with autism spectrum disorder. Biological Psychiatry, 78(9), 625-634. https://doi.org/10.1016/j. biopsych.2015.03.024

Langen, M., Bos, D., Noordermeer, S. D., Nederveen, H., van Engeland, H., \& Durston, S. (2014). Changes in the development of striatum are involved in repetitive behavior in autism. Biological Psychiatry, 76(5), 405-411. https://doi.org/10.1016/j.biopsych.2013.08.013

Lench, D. H., DeVries, W., \& Hanlon, C. A. (2017). The effect of task difficulty on motor performance and frontal-striatal connectivity in cocaine users. Drug and Alcohol Dependence, 173, 178-184. https:// doi.org/10.1016/j.drugalcdep.2016.12.008

Lidstone, D. E., Miah, F. Z., Poston, B., Beasley, J. F., Mostofsky, S. H., \& Dufek, J. S. (2020). Children with autism spectrum disorder show impairments during dynamic versus static grip-force tracking. Autism Research, 13(12), 2177-2189. https://doi.org/10.1002/aur.2370

Lord, C., Rutter, M., Goode, S., Heemsbergen, J., Jordan, H., Mawhood, L., \& Schopler, E. (1989). Autism diagnostic observation schedule: A standardized observation of communicative and social behavior. Journal of Autism and Developmental Disorders, 19(2), 185-212.

Lord, C., Rutter, M., \& Le Couteur, A. (1994). Autism diagnostic interviewrevised: A revised version of a diagnostic interview for caregivers of individuals with possible pervasive developmental disorders. Journal of Autism and Developmental Disorders, 24(5), 659-685.

MacNeil, L. K., \& Mostofsky, S. H. (2012). Specificity of dyspraxia in children with autism. Neuropsychology, 26(2), 165-171. https://doi.org/ 10.1037/a0026955

McKinney, W. S., Bartolotti, J., Khemani, P., Wang, J. Y., Hagerman, R. J., \& Mosconi, M. W. (2020). Cerebellar-cortical function and connectivity during sensorimotor behavior in aging FMR1 gene premutation carriers. Neuroimage Clinical, 27, 102332. https://doi.org/10.1016/j.nicl. 2020.102332

McKinney, W. S., Wang, Z., Kelly, S., Khemani, P., Lui, S., White, S. P., \& Mosconi, M. W. (2019). Precision sensorimotor control in aging FMR1 gene premutation carriers. Frontiers in Integrative Neuroscience, 13, 56. https://doi.org/10.3389/fnint.2019.00056

McLaren, D. G., Ries, M. L., Xu, G., \& Johnson, S. C. (2012). A generalized form of context-dependent psychophysiological interactions (gPPI): A comparison to standard approaches. Neurolmage, 61(4), 1277-1286. https://doi.org/10.1016/j.neuroimage.2012.03.068

Minshew, N. J., Sweeney, J., \& Luna, B. (2002). Autism as a selective disorder of complex information processing and underdevelopment of neocortical systems. Molecular Psychiatry, 7(2), S14-S15. https://doi.org/ 10.1038/sj.mp.4001166

Morimoto, C., Hida, E., Shima, K., \& Okamura, H. (2018). Temporal processing instability with millisecond accuracy is a cardinal feature of sensorimotor impairments in autism spectrum disorder: Analysis using the synchronized finger-tapping task. Journal of Autism and Developmental Disorders, 48(2), 351-360. https://doi.org/10.1007/s10803017-3334-7

Mosconi, M. W., Kay, M., D'Cruz, A. M., Guter, S., Kapur, K., Macmillan, C., ... Sweeney, J. A. (2010). Neurobehavioral abnormalities in first-degree relatives of individuals with autism. Archives of General Psychiatry, 67(8), 830-840. https://doi.org/10.1001/archgenpsychiatry.2010.87

Mosconi, M. W., Mohanty, S., Greene, R. K., Cook, E. H., Vaillancourt, D. E., \& Sweeney, J. A. (2015). Feedforward and feedback motor control abnormalities implicate cerebellar dysfunctions in autism spectrum disorder. The Journal of Neuroscience, 35(5), 20152025. https://doi.org/10.1523/JNEUROSCI.2731-14.2015

Mostofsky, S. H., Powell, S. K., Simmonds, D. J., Goldberg, M. C., Caffo, B., \& Pekar, J. J. (2009). Decreased connectivity and cerebellar activity in autism during motor task performance. Brain, 132(Pt 9), 2413-2425. https://doi.org/10.1093/brain/awp088

Muller, R. A., Kleinhans, N., Kemmotsu, N., Pierce, K., \& Courchesne, E. (2003). Abnormal variability and distribution of functional maps in autism: An FMRI study of visuomotor learning. The American Journal of Psychiatry, 160(10), 1847-1862. https://doi.org/10.1176/appi.ajp. 160.10.1847

Muller, R. A., Pierce, K., Ambrose, J. B., Allen, G., \& Courchesne, E. (2001). Atypical patterns of cerebral motor activation in autism: A functional magnetic resonance study. Biological Psychiatry, 49(8), 665-676.

Nebel, M. B., Eloyan, A., Nettles, C. A., Sweeney, K. L., Ament, K., Ward, R. E., ... Mostofsky, S. H. (2016). Intrinsic visual-motor synchrony correlates with social deficits in autism. Biological Psychiatry, 79(8), 633-641. https://doi.org/10.1016/j.biopsych.2015.08.029

Neely, K. A., Mohanty, S., Schmitt, L. M., Wang, Z., Sweeney, J. A., \& Mosconi, M. W. (2019). Motor memory deficits contribute to motor impairments in autism spectrum disorder. Journal of Autism and Developmental Disorders, 49(7), 2675-2684.

Padmanabhan, A., Lynn, A., Foran, W., Luna, B., \& O'Hearn, K. (2013). Age related changes in striatal resting state functional connectivity in autism. Frontiers in Human Neuroscience, 7, 814. https://doi.org/10. 3389/fnhum.2013.00814

Paus, T. (2001). Primate anterior cingulate cortex: Where motor control, drive and cognition interface. Nature Reviews. Neuroscience, 2(6), 417424. https://doi.org/10.1038/35077500

Pincus, S. M., \& Goldberger, A. L. (1994). Physiological time-series analysis: What does regularity quantify? The American Journal of Physiology, 266(4 Pt 2), H1643-H1656. https://doi.org/10.1152/ajpheart.1994. 266.4. $\mathrm{H} 1643$

Pool, E. M., Rehme, A. K., Fink, G. R., Eickhoff, S. B., \& Grefkes, C. (2014). Handedness and effective connectivity of the motor system. Neurolmage, 99, 451-460. https://doi.org/10.1016/j.neuroimage. 2014.05.048

Prodoehl, J., Yu, H., Wasson, P., Corcos, D. M., \& Vaillancourt, D. E. (2008). Effects of visual and auditory feedback on sensorimotor circuits in the basal ganglia. Journal of Neurophysiology, 99(6), 3042-3051. https:// doi.org/10.1152/jn.01108.2007

Ramos, T. C., Balardin, J. B., Sato, J. R., \& Fujita, A. (2018). Abnormal cortico-cerebellar functional connectivity in autism spectrum disorder. Frontiers in Systems Neuroscience, 12, 74. https://doi.org/10.3389/ fnsys.2018.00074

Reilly, J. L., Harris, M. S., Keshavan, M. S., \& Sweeney, J. A. (2005). Abnormalities in visually guided saccades suggest corticofugal dysregulation in never-treated schizophrenia. Biological Psychiatry, 57(2), 145-154. https://doi.org/10.1016/j.biopsych.2004.10.024

Rypma, B., Prabhakaran, V., Desmond, J. E., Glover, G. H., \& Gabrieli, J. D. (1999). Load-dependent roles of frontal brain regions in the maintenance of working memory. Neurolmage, 9(2), 216-226. https://doi. org/10.1006/nimg.1998.0404

Sack, A. T., Sperling, J. M., Prvulovic, D., Formisano, E., Goebel, R., Di Salle, F., ... Linden, D. E. (2002). Tracking the mind's image in the brain II: Transcranial magnetic stimulation reveals parietal asymmetry in visuospatial imagery. Neuron, 35(1), 195-204. https://doi.org/10. 1016/s0896-6273(02)00745-6

Schmitt, L. M., Bojanek, E., White, S. P., Ragozzino, M. E., Cook, E. H., Sweeney, J. A., \& Mosconi, M. W. (2019). Familiality of behavioral flexibility and response inhibition deficits in autism spectrum disorder (ASD). Molecular Autism, 10, 47. https://doi.org/10.1186/s13229019-0296-y

Schmitt, L. M., Cook, E. H., Sweeney, J. A., \& Mosconi, M. W. (2014). Saccadic eye movement abnormalities in autism spectrum disorder indicate dysfunctions in cerebellum and brainstem. Molecular Autism, 5(1), 47. https://doi.org/10.1186/2040-2392-5-47

Schnell, K., Heekeren, K., Schnitker, R., Daumann, J., Weber, J., Hesselmann, V., ... Gouzoulis-Mayfrank, E. (2007). An fMRI approach 
to particularize the frontoparietal network for visuomotor action monitoring: Detection of incongruence between test subjects' actions and resulting perceptions. Neurolmage, 34(1), 332-341. https://doi.org/10. 1016/j.neuroimage.2006.08.027

Sharer, E., Crocetti, D., Muschelli, J., Barber, A. D., Nebel, M. B., Caffo, B. S., ... Mostofsky, S. H. (2015). Neural correlates of visuomotor learning in autism. Journal of Child Neurology, 30(14), 1877-1886. https://doi.org/10.1177/0883073815600869

Slifkin, A. B., \& Newell, K. M. (1999). Noise, information transmission, and force variability. Journal of Experimental Psychology. Human Perception and Performance, 25(3), 837-851.

Slifkin, A. B., Vaillancourt, D. E., \& Newell, K. M. (2000). Intermittency in the control of continuous force production. Journal of Neurophysiology, 84(4), 1708-1718. https://doi.org/10.1152/jn.2000.84.4.1708

Spraker, M. B., Corcos, D. M., Kurani, A. S., Prodoehl, J., Swinnen, S. P., \& Vaillancourt, D. E. (2012). Specific cerebellar regions are related to force amplitude and rate of force development. Neurolmage, 59(2), 1647-1656. https://doi.org/10.1016/j.neuroimage.2011.09.019

Stein, J. F., \& Glickstein, M. (1992). Role of the cerebellum in visual guidance of movement. Physiological Reviews, 72(4), 967-1017.

Stoodley, C. J., D'Mello, A. M., Ellegood, J., Jakkamsetti, V., Liu, P., Nebel, M. B., ... Tsai, P. T. (2017). Altered cerebellar connectivity in autism and cerebellar-mediated rescue of autism-related behaviors in mice. Nature Neuroscience, 20(12), 1744-1751. https://doi.org/10. 1038/s41593-017-0004-1

Stoodley, C. J., \& Schmahmann, J. D. (2009). Functional topography in the human cerebellum: A meta-analysis of neuroimaging studies. Neurolmage, 44(2), 489-501. https://doi.org/10.1016/j.neuroimage. 2008.08.039

Takarae, Y., Minshew, N. J., Luna, B., \& Sweeney, J. A. (2004). Oculomotor abnormalities parallel cerebellar histopathology in autism. Journal of Neurology, Neurosurgery, and Psychiatry, 75(9), 1359-1361. https:// doi.org/10.1136/jnnp.2003.022491

Takarae, Y., Minshew, N. J., Luna, B., \& Sweeney, J. A. (2007). Atypical involvement of frontostriatal systems during sensorimotor control in autism. Psychiatry Research, 156(2), 117-127. https://doi.org/10. 1016/j.pscychresns.2007.03.008

Tsakiris, M., Longo, M. R., \& Haggard, P. (2010). Having a body versus moving your body: Neural signatures of agency and body-ownership. Neuropsychologia, 48(9), 2740-2749. https://doi.org/10.1016/j. neuropsychologia.2010.05.021

Unruh, K. E., Martin, L. E., Magnon, G., Vaillancourt, D. E., Sweeney, J. A., \& Mosconi, M. W. (2019). Cortical and subcortical alterations associated with precision visuomotor behavior in individuals with autism spectrum disorder. Journal of Neurophysiology, 122(4), 1330-1341. https://doi.org/10.1152/jn.00286.2019

Unruh, K. E., McKinney, W. S., Bojanek, E. K., Fleming, K. K., Sweeney, J. A., \& Mosconi, M. W. (2021). Initial action output and feedback-guided motor behaviors in autism spectrum disorder. Molecular Autism, 12(1), 52. https://doi.org/10.1186/s13229-021-00452-8

Vaillancourt, D. E., Haibach, P. S., \& Newell, K. M. (2006). Visual angle is the critical variable mediating gain-related effects in manual control.
Experimental Brain Research, 173(4), 742-750. https://doi.org/10. 1007/s00221-006-0454-2

Vaillancourt, D. E., Mayka, M. A., \& Corcos, D. M. (2006). Intermittent visuomotor processing in the human cerebellum, parietal cortex, and premotor cortex. Journal of Neurophysiology, 95(2), 922-931. https:// doi.org/10.1152/jn.00718.2005

Vaillancourt, D. E., Slifkin, A. B., \& Newell, K. M. (2001). Regularity of force tremor in Parkinson's disease. Clinical Neurophysiology, 112(9), 15941603. https://doi.org/10.1016/s1388-2457(01)00593-4

Villalobos, M. E., Mizuno, A., Dahl, B. C., Kemmotsu, N., \& Muller, R. A. (2005). Reduced functional connectivity between V1 and inferior frontal cortex associated with visuomotor performance in autism. Neurolmage, 25(3), 916-925. https://doi.org/10.1016/j.neuroimage. 2004.12.022

Wang, Z., Hallac, R. R., Conroy, K. C., White, S. P., Kane, A. A., Collinsworth, A. L., ... Mosconi, M. W. (2016). Postural orientation and equilibrium processes associated with increased postural sway in autism spectrum disorder (ASD). Journal of Neurodevelopmental Disorders, 8, 43. https://doi.org/10.1186/s11689-016-9178-1

Wang, Z., Magnon, G. C., White, S. P., Greene, R. K., Vaillancourt, D. E., \& Mosconi, M. W. (2015). Individuals with autism spectrum disorder show abnormalities during initial and subsequent phases of precision gripping. Journal of Neurophysiology, 113(7), 1989-2001. https://doi. org/10.1152/jn.00661.2014

Wang, Z., Wang, Y., Sweeney, J. A., Gong, Q., Lui, S., \& Mosconi, M. W. (2019). Resting-state Brain network dysfunctions associated with visuomotor impairments in autism Spectrum disorder. Frontiers in Integrative Neuroscience, 13, 17. https://doi.org/10.3389/fnint.2019. 00017

Wechsler, D. (1999). Wechsler abbreviated scale of intelligence (WASI). New York, NY: The Psychological Corporation: Harcourt Brace \& Company.

Yim, M. Y., Aertsen, A., \& Kumar, A. (2011). Significance of input correlations in striatal function. PLoS Computational Biology, 7(11), e1002254. https://doi.org/10.1371/journal.pcbi.1002254

\section{SUPPORTING INFORMATION}

Additional supporting information may be found in the online version of the article at the publisher's website.

How to cite this article: Lepping, R. J., McKinney, W. S., Magnon, G. C., Keedy, S. K., Wang, Z., Coombes, S. A., Vaillancourt, D. E., Sweeney, J. A., \& Mosconi, M. W. (2022). Visuomotor brain network activation and functional connectivity among individuals with autism spectrum disorder. Human Brain Mapping, 43(2), 844-859. https://doi.org/10. 1002/hbm.25692 